ISSN: 0213-2087 eISSN: 2444-7080

DOI: https://doi.org/10.14201/shhc202139269302

\title{
EL DESARROLLO DE LA RELACIÓN CHINA- FRANCIA: DEL RECONOCIMIENTO MUTUO A LA PROYECCIÓN DE LA INICIATIVA CINTURÓN Y RUTA DE LA SEDA
}

\section{The development of the China-France relationship: from mutual recognition to the projection of the Belt and Road Initiative}

\author{
Manuel de Jesús ROCHA PINO* \\ Universidad Nacional Autónoma de México \\ manuelrocha@politicas.unam.mx
}

https://orcid.org/0000-0002-7295-9056

Recibido: 17/05/2021 Revisado: 16/07/2021 Aceptado: 19/07/2021

RESUMEN: En la investigación se analizan los principales elementos estructurales que ha desarrollado la relación sino-francesa a partir de sus políticas de reconocimiento mutuo, así como la percepción en Francia sobre la instrumentación de la Iniciativa Cinturón y Ruta de la Seda en su territorio. Desde el establecimiento de sus relaciones diplomáticas en 1964, la relación sino-francesa ha sido instrumentada de acuerdo con algunos intereses nacionales que han definido sus características estructurales: 1) el interés de Francia por mantener una relación "privilegiada" con China en el contexto europeo; 2) el interés por mantener relaciones comerciales activas; 3) el interés de China por ser reconocida por una potencia occidental (económica, cultural, nuclear y perteneciente al Consejo de Seguridad de Naciones Unidas); 4) el interés

* Investigador adscrito a la Facultad de Ciencias Políticas y Sociales (FCPyS) de la Universidad Nacional Autónoma de México. Becario del Programa de Becas Posdoctorales en la unAm. El autor agradece a la Dra. Yleana Cid Capetillo y al Dr. Alejandro Chanona Burguete de la FCPyS, así como a dos dictaminadores anónimos por sus sugerencias. Todos los errores y omisiones corresponden al autor. 
de Francia por ser una orientadora de las políticas de la Unión Europea hacia China. En la investigación se identifica cómo estas características han sido instrumentalizadas por los distintos gobiernos de la República Francesa y la percepción generada por los gobiernos de la República Popular China.

Palabras clave: asociación estratégica China-Francia; multipolaridad; Guerra Fría; Posguerra Fría; derechos humanos; Iniciativa Cinturón y Ruta de la Seda.

ABSTRACT: This research analyzes the main structural elements that the SinoFrench relationship has developed from its policies of mutual recognition, as well as the perception in France about the implementation of the Belt and Silk Road Initiative into French territory. Since the establishment of diplomatic relations in 1964, the Sino-French relationship has been implemented in accordance with some national interests that have defined its structural characteristics: 1) France's interest about maintaining a "privileged" relationship with China in the European context; 2) the interest about maintaining active trade relations; 3) China's interest about having diplomatic relations with a Western Great Power (on economics, culture, nuclear capacity and a UN Security Council's member); 4) France's interest about being a guide for the European Union's policies towards China. The research identifies how these characteristics have been instrumental for the different governments of the French Republic and the perception generated by the governments of the People's Republic of China.

Key words: China-France strategic partnership; multipolarity; Cold War; Postcold War; Human Rights; Belt and Silk Road Initiative.

\section{INTRODUCCIÓN}

En la investigación se analiza el desarrollo histórico de los vínculos políticos y económicos mantenidos en la relación bilateral China-Francia desde que ambos países establecieron relaciones diplomáticas en 1964 hasta el año 2021. Especialmente son consideradas las dinámicas de cooperación que han desarrollado como parte de la agenda de la asociación estratégica China-Francia establecida desde 1997, así como los proyectos que China ha instrumentado en territorio francés como parte de la Iniciativa Cinturón y Ruta de la Seda.

Como marco teórico, la investigación utiliza un modelo de análisis basado en el realismo neoclásico de Relaciones Internacionales para examinar los instrumentos, diplomáticos e institucionales, implementados por los gobiernos chino y francés para incentivar sus mecanismos de diálogo y cooperación. En la investigación se considera que desde el momento de la normalización de sus relaciones, China y Francia han instrumentado sus políticas de aproximación mediante consideraciones estratégicas sustentadas en sus intereses nacionales orientadas por factores internos y la percepción de sus dirigencias nacionales aunque, por lo general, limitadas por la estructura del statu quo internacional vigente en cada contexto histórico. Como menciona Víctor M. Mijares, el realismo neoclásico: 


\section{MANUEL DE JESÚS ROCHA PINO \\ EL DESARROLLO DE LA RELACIÓN CHINA-FRANCIA: DEL RECONOCIMIENTO MUTUO 271 A LA PROYECCIÓN DE LA INICIATIVA CINTURÓN Y RUTA DE LA SEDA}

Asume que la distribución de capacidades en el sistema internacional (variable independiente) genera particulares tendencias en las políticas exteriores (variable dependiente), pero que estas varían de caso en caso en conformidad con las percepciones, capacidades y operatividad doméstica del «ejecutivo de política exterior» (EPE) (variable interviniente) ${ }^{1}$.

Como señalan Ripsman, Taliaferro y Lobell para el modelo de análisis del realismo neoclásico existen un conjunto de «variables intervinientes» como la política interior de los actores, las percepciones de los líderes estatales, la cultura estratégica, las relaciones estado-sociedad y los acuerdos institucionales internos para explicar el proceso de la toma de decisiones de la política exterior: «estas variables incluyen modelos psicológicos, burocrático-organizacionales, societales e institucionales, los cuales reflejan aproximaciones alternativas de análisis de la política exterior ${ }^{2}$.

Desde el momento en que Francia y China iniciaron relaciones diplomáticas, han mantenido algunas prioridades definidas por ambos actores, compartidas de una manera asimétrica: 1) el interés de ambas por mantener el flujo de sus intercambios comerciales; 2) el interés de Francia por situarse como un interlocutor privilegiado de China en Europa; 3) el interés de China por contar con el reconocimiento diplomático francés al mismo tiempo que mantiene una relación estrecha con una de las principales economías de la Unión Europea (UE) y con una potencia nuclear europea; 4) el desarrollo de un diálogo bilateral estructurado con diferentes matices de acuerdo con el contexto histórico: durante la Guerra Fría, como una posición intermedia frente a la conducta hegemónica de las grandes potencias (la Unión Soviética y Estados Unidos, en adelante EEUU). Posteriormente, durante la Posguerra Fría, el diálogo bilateral sino-francés ha tratado de fomentar su cooperación en los mecanismos multilaterales. A lo largo de las diferentes coyunturas, los líderes nacionales de Francia y China han desarrollado percepciones particulares que han definido el curso de la relación bilateral como variables intervinientes.

Como ha ocurrido con otras relaciones bilaterales de China con actores de Europa occidental (incluida la UE en el nivel supranacional), Francia favorece la preferencia por sostener, de manera activa, un diálogo sobre temas normativos con Pekín (como la situación de los derechos humanos, el Estado de Derecho y la democratización del sistema político chino). Sin embargo, los distintos gobiernos que han presidido a la República Francesa han mostrado en diferentes momentos una interpretación particular sobre la instrumentación de una agenda normativa (en ocasiones dependiendo de la conflictividad global existente y de la alineación

1. Mujares, Víctor M.: «Realismo neoclásico: ¿El retorno de los estudios internacionales a la ciencia política?», Revista de Ciencia Política, 35(3), 2015, p. 582.

2. Ripsman, Norrin M.; TAliaferro, Jeffrey W. y Lobell, Steven E.: «Neoclassical Realist Intervening Variables». En: Ripsman, Norrin M., et al. (ed.): Neoclassical Realist Theory of International Politics. New York: Oxford University Press, 2016, pp. 58-59. 
MANUEL DE JESÚS ROCHA PINO

EL DESARROLLO DE LA RELACIÓN CHINA-FRANCIA: DEL RECONOCIMIENTO MUTUO A LA PROYECCIÓN DE LA INICIATIVA CINTURÓN Y RUTA DE LA SEDA

de las políticas de Francia hacia Pekín con las ejercidas por otras potencias como EEUU).

Además de las secciones de la Introducción y las Conclusiones, la investigación se divide en las siguientes partes: en la segunda, que abarca el periodo 19641995, se elabora una descripción de los inicios de la relación China-Francia a lo largo de la Guerra Fría y los primeros años de la Posguerra Fría: de esta manera se identifican los rasgos estructurales que serán definitorios para la conformación del vínculo de asociación estratégica sino-francés de 1997.

En la tercera, se analiza la trayectoria de la relación sino-francesa durante el gobierno de Jacques Chirac (1995-2007) contextualizada en el periodo de Posguerra Fría. Durante el gobierno de Chirac, las prioridades bilaterales se orientaron hacia las relaciones comerciales y la cooperación en asuntos políticos y culturales. Como resultado en 1997 se acordó el establecimiento de la asociación estratégica China-Francia y, en 2003, ambos actores efectuaron un acercamiento diplomático en los ámbitos bilateral y multilateral, a partir de las críticas de China, Francia, Alemania y otros actores a la intervención de EEUU en Irak.

En la cuarta, se analiza la reaproximación efectuada por el gobierno de Nicolas Sarzoky (2007-2012) con el de George W. Bush a partir de 2007. La reaproximación transatlántica llevó al gobierno de Sarkozy a considerar una nueva definición de los términos de la asociación estratégica global China-Francia (una acción rechazada por Pekín y que provocó un deterioro en la relación bilateral). Sin embargo, las tensiones se modificaron con el inicio de la crisis económica mundial en 2008: a partir de 2009-2010, la relación sino-francesa se encaminó hacia el pragmatismo económico (coincidiendo con una postura similar en la relación China-UE).

En la quinta, se analiza la trayectoria de la relación sino-francesa durante los gobiernos de François Hollande (2012-2017) y Emmanuel Macron (2017). Este periodo se ha caracterizado por la continuación de la aproximación pragmática, incluyendo la instrumentación de medidas para mejorar la confianza en la relación durante el gobierno de Hollande (debido al distanciamiento ocurrido durante el periodo de Sakozy). A partir de 2014 se presentó la Iniciativa Cinturón y Ruta de la Seda en el espacio de la uE y en Francia se han desarrollado algunos de sus proyectos de inversión, preferentemente enfocados en la modernización de la infraestructura portuaria y de comunicaciones, así como en inversiones de capital chino en activos industriales y cooperación financiera (en 2015 Francia se adhirió como miembro fundador del Banco Asiático de Inversión en Infraestructura - BAII).

En la investigación se trata de definir el carácter estructural o coyuntural de las relaciones de cooperación mantenidas durante las diversas etapas desarrolladas en la relación China-Francia durante el espacio de tiempo analizado, así como su capacidad para compartir preferencias y evitar las limitantes impuestas por otros actores como EEUU. 


\section{MANUEL DE JESÚS ROCHA PINO \\ EL DESARROLLO DE LA RELACIÓN CHINA-FRANCIA: DEL RECONOCIMIENTO MUTUO 273 A LA PROYECCIÓN DE LA INICIATIVA CINTURÓN Y RUTA DE LA SEDA}

\section{Las relaciones Francia-República Popular China: del reconocimiento mutuo A LAS TENSIONES EN MATERIA NORMATIVA (1964-1995)}

\subsection{La normalización de relaciones diplomáticas entre China y Francia (1964)}

Con el establecimiento de relaciones diplomáticas entre China y Francia, en enero de 1964, el gobierno de Charles de Gaulle convirtió a Francia en uno de los primeros países miembros de la Organización del Tratado del Atlántico Norte (OTAN) en impulsar una política de reconocimiento mutuo con la República Popular aunque, como antecedente, el Reino Unido había establecido relaciones con China en 1950 debido a la necesidad por mantener la estabilidad en su colonia de Hong Kong y a los intereses económicos británicos en otras regiones como Shanghai y Guangzhou (aunque en el caso de la relación sino-británica, el intercambio bilateral de embajadores ocurriría hasta 1972) ${ }^{3}$.

Al normalizar sus relaciones con el gobierno maoísta, el Presidente de Gaulle trataba de alcanzar varios objetivos coyunturales: 1) mostrar una independencia hacia la política exterior estadounidense, especialmente luego de que en 1963 fuera acordado en Moscú el Tratado de Prohibición Parcial de Ensayos Nucleares entre eEuu, el Reino Unido y la Unión Soviética (Limited Test Ban Treaty - LTBT, por sus siglas en inglés) pero sin la participación de París (por su lado, en 1963 Francia vetó la solicitud de adhesión del Reino Unido a la Comunidad Económica Europea - CEE) $;{ }^{4}$ 2) aproximarse a China en un momento que Francia desocupaba a sus colonias en Asia y África y la situación en Vietnam del Sur se hacía más inestable (Pekín y París habían aproximado sus críticas hacia el régimen de Ngo Dinh Diem en Saigón); y 3) fomentar las relaciones económico-comerciales sino-francesas 5 . Así mismo, el final de la Guerra de Liberación en Argelia en 1962 excluyó un obstáculo para la aproximación bilateral ${ }^{6}$.

El proceso de negociación para el establecimiento de las relaciones bilaterales sino-francesas ocurrió en un momento singular de la Guerra Fría: además de un

3. GARRET, Martin: «Playing the China Card? Revisiting France's Recognition of Communist China, 1963-1964", Journal of Cold War Studies, 10(1), 2008, p. 52.

4. LIU, Kaixuan: "Les relations politiques francochinoises de 1949 à 1983: entre mythe et réalité", Monde Chinois, 59(3), 2019, p. 18.

El Tratado de Prohibición Parcial de Ensayos Nucleares de 1963 prohibía la realización de pruebas con armas nucleares en la atmósfera, bajo la superficie del agua y en el espacio. Francia y China no firmaron el acuerdo y continuaron haciendo pruebas nucleares hasta 1996 (en ese año ambos países se sumaron al Tratado de Prohibición Completa de los Ensayos Nucleares). Los últimos ensayos nucleares subterráneos por parte de China se efectuaron en Lop Nor, Xinjiang. Francia realizó sus últimos ensayos nucleares submarinos en los atolones de Mururoa y Fangataufa en la Polinesia Francesa. Ver: NorRIS, Robert S.: "French and Chinese Nuclear Weapon Testing», Security Dialogue, 27(1), 1996, pp. 43-46.

5. GARRET, Martin, op. cit., p. 60; LüTHI, Lorenz: «Rearranging International Relations? How Mao's China and de Gaulle's France Recognized Each Other in 1963-1964", Journal of Cold War Studies, 16(1), 2014, pp. 112-113.

6. Liu, Kaixuan, op. cit., p. 18. 
distanciamiento franco-estadounidense, provocado por acciones percibidas desde París como contrarias a sus intereses y su posición como Gran Potencia (en el aspecto estratégico, con acciones como la firma tripartita del LTBT), también deben tomarse en cuenta las consecuencias de la ruptura entre China y la Unión Soviética a partir de 1960, en un momento en que el poder del Presidente del Partido Comunista Chino -PCCh- Mao Zedong, para dirigir las políticas económicas de China, había disminuido en favor de las decisiones del Presidente de la República Popular, Liu Shaoqi, y todavía no iniciaban las movilizaciones de la Revolución Cultural de $1966^{7}$.

Así mismo, Francia buscaba incrementar sus relaciones económicas con China. Este interés había comenzado desde 1956 con la visita de representantes de negocios franceses a Pekín, en un sentido contrario a las políticas de sanciones y embargos llevados a cabo por Washington en su contra (en ese momento era claro el superávit comercial francés frente a la economía china) ${ }^{8}$. Por su lado, China también mostraba interés en contar con relaciones económicas que superaran el embargo estadounidense. Por esta razón, los contactos sino-franceses para dialogar de forma abierta sobre un establecimiento de relaciones se realizaron de manera discreta y en espacios neutrales: a mediados de 1962 asistió a Ginebra el entonces embajador chino en Polonia, Wang Bingnan, para reunirse con los funcionarios del consulado francés en dicha ciudad y abordar el tema?.

Posteriormente, el embajador francés en Suiza comunicó a la embajada china en Berna el interés de su gobierno por enviar una delegación comercial a China, una propuesta que fue aceptada por Pekín ${ }^{10}$ : entre 1962-1963, China había importado productos agrícolas desde Francia, como cereales, debido a las malas cosechas que siguieron a las consecuencias de la colectivización del Gran Salto Adelante $^{11}$. Las conversaciones secretas continuaron en Berna a lo largo de 1963 y entre octubre-noviembre del mismo año, asistió a Pekín el antiguo Primer Ministro francés, Edgar Faure, para continuar con la negociación: el 27 de enero de 1964, París anunció mediante un "corto comunicado" el establecimiento de relaciones con China $^{12}$. En el caso de la relación sino-francesa el intercambio de embajadores fue inmediato ${ }^{13}$.

7. Garret, Martin, op. cit., p. 63; Lieberthal, Kenneth: «The Great Leap Foreward and the Split in the Yenan Leadership». En: Macfarquhar, Roderick y Fairbank, John (eds.): The Cambridge History of China vol. 14. The People's Republic Part 1: The Emergence of Revolutionary China. Cambridge: Cambridge University Press, 1987, pp. 326-327.

8. Robin, Thierry: «France facing the People's Republic of China (1949-1964). A Policy of Economic Relations Under Control», Sinica Venetiana, 5, 2018, pp. 127-128.

9. LÜTHI, Lorenz, op. cit., p. 120.

10. Ibidem. p. 121. RoBin, Thierry op. cit., pp. 133-134.

11. Ibidem. p. 132-133.

12. LüTHI, Lorenz, op. cit., p. 112.

13. Ibidem. p. 129. 


\section{MANUEL DE JESÚS ROCHA PINO \\ EL DESARROLLO DE LA RELACIÓN CHINA-FRANCIA: DEL RECONOCIMIENTO MUTUO 275 A LA PROYECCIÓN DE LA INICIATIVA CINTURÓN Y RUTA DE LA SEDA}

China se interesó en contar con el reconocimiento de un aliado occidental y fomentar las relaciones con un país relevante en Europa como Francia, además miembro permanente del Consejo de Seguridad de la Organización de las Naciones Unidas (ONU), en un momento en que la ruptura sino-soviética desembocaba en mayores disputas ideológicas e incluso estratégicas (un contexto que era tomado en cuenta por el gobierno de De Gaulle, quien evaluó que el maoísmo buscaría «acomodarse» a las políticas de los países de Europa occidental) ${ }^{14}$. La aproximación sino-francesa propició que el maoísmo iniciara una relación incipiente con el proyecto de integración de la entonces Comunidad Económica Europea (CEe): desde una perspectiva realista, Pekín desarrolló la percepción de que el proceso de integración podía presentarse como una alternativa de equilibrio hacia la hegemonía ejercida por la bipolaridad soviético-estadounidense ${ }^{15}$.

Desde la perspectiva del maoísmo, la posición de la cEe fue estimada en una "zona intermedia" o como una "coalición de gobiernos" (y no como una "coalición de monopolio del capital») de acuerdo con el concepto de las Zonas Intermedias (los países posicionados entre las dos potencias hegemónicas) correspondiente a la Teoría de los Tres Mundos de Mao Zedong (quien había comenzado a formular esta teoría al final de la década de 1940) ${ }^{16}$. La Teoría de los Tres Mundos de Mao dividía al sistema internacional en tres categorías: el Primer Mundo lo constituían las potencias hegemónicas: EEuU y la Unión Soviética. El Segundo Mundo lo integraban los países desarrollados como los de Europa Occidental, Japón, Australia y Canadá; y el Tercer Mundo, los países en vías de desarrollo de África, Asia y América Latina (incluida China) ${ }^{17}$. A su vez, como menciona Jean-Yves Haine, para el Presidente de Gaulle «el círculo atlántico solo podía significar subordinación [...] como su periodo presidencial demostró, la búsqueda de la igualdad, es decir, la soberanía y, por lo tanto, la autonomía, fue a menudo difícil, siempre costosa y regularmente incomprendida " ${ }^{18}$. El rechazo a la dependencia en un mundo bipolar provocó la atracción inicial de la presidencia francesa hacia el concepto chino sobre la multipolaridad.

De acuerdo con la percepción maoísta, los seis países que integraban a la CEE (en este caso representados por Francia) estaban inconformes con EEuU, mientras

14. GARRET, Martin, op. cit., p. 61.

15. Ibidem. p. 62.

16. Idem; Liu, Lirong: "The Evolution of China's Eu Policy: from Mao's Intermediate Zone to a Strategic Partnership based on non-shared Values", Journal of European Integration History, 18, 2012, pp. 12-16.

17. Como menciona Liu Lirong "la teoría de los Tres Mundos facilitó la cooperación política y económica entre China y los países de Europa Occidental. La teoría de las Zonas Intermedias y la Teoría de los Tres Mundos se basan en la visión del mundo multipolar» Ver: Ibidem. p. 16; MAO, Zedong: "On the Question of the Differentiation of the Three Worlds». En: MINISTERIO DE ASUNTOS EXTERIORES DE CHINA (comp.): On Diplomacy. Pekín: Foreign Languages Press, 1998, p. 454, en: http://michaelharrison.org. uk/wp-content/uploads/2017/02/Mao-Zedong-On-Diplomacy-1998.pdf.

18. Haine, Jean-Yves: "A New Gaullist Moment? European Bandwagoning and International Polarity", International Affairs, 95(5), 2015, pp. 1002-1003. 
MANUEL DE JESÚS ROCHA PINO

EL DESARROLLO DE LA RELACIÓN CHINA-FRANCIA: DEL RECONOCIMIENTO MUTUO

A LA PROYECCIÓN DE LA INICIATIVA CINTURÓN Y RUTA DE LA SEDA

algunos países de Europa Oriental (como Albania y anteriormente Hungría) mostraban una inconformidad hacia el liderazgo soviético ejercido por Nikita Kruschev ${ }^{19}$. Como parte de la Teoría de los Tres Mundos se agregaba la interpretación de Mao del concepto de multipolaridad, el cual tuvo algunas similitudes, a su vez, con la percepción gaullista sobre la construcción de un mundo multipolar; como menciona Garret: "de Gaulle entendió que el cambio en el equilibrio global se produciría solo a largo plazo. No obstante, esperaba que su política hacia la República Popular China demostrara ser un paso importante en el surgimiento de un mundo más multipolar»: la multipolaridad se opondría a la hegemonía de las dos potencias ${ }^{20}$. En este momento de la relación sino-francesa, durante el contexto de la no subordinación a las potencias hegemónicas, ambos países hicieron coincidir fragmentos de su crítica al sistema internacional de la Guerra Fría mediante la aproximación de la percepción de de Gaulle sobre el mundo multipolar y el concepto maoísta de la multipolaridad. Aunque este cambio en los intereses de los actores fue limitado (Francia siguió comprometida con sus intereses e identidad como parte de la Alianza Atlántica y China mantendría su compromiso ideológico con los movimientos de liberación que tomaban como referencia a la revolución maoísta y a la Guerra Popular).

Una cuestión que facilitó los acercamientos fue que Francia había abandonado sus concesiones en los Puertos de Tratado en China desde 1946, entre las que habían sobresalido las ubicadas en Shanghai, Tianjin y Kunming (en Yunnan, una provincia china fronteriza con la antigua Indochina francesa), por lo que el reconocimiento mutuo ocurrió sin disputas territoriales recientes: el recuerdo de Francia como una potencia colonial en China fue mitigado debido a que, durante la Segunda Guerra Mundial, las autoridades de la Francia Libre se habían comprometido a reconocer la autoridad de la entonces República de China en las antiguas concesiones francesas al terminar la Guerra (ocupadas por el Imperio del Japón $)^{21}$.

El principal inconveniente en el acercamiento sino-francés en 1964 ocurrió relacionado con la cuestión del Estrecho de Taiwán. Para el gobierno francés el establecimiento de relaciones con China podría concretarse sin necesidad de romper relaciones con la República de China, exiliada entonces en Taiwán: en respuesta, Pekín comunicó que para oficializar a la relación sino-francesa era una

19. LüTHI, Lorenz, op. cit., p. 121.

20. Garret, Martin, op. cit., pp. 61-62; Hou, Qibin: Quarante ans de dialogue: évolution des relations politico-diplomatiques entre la France et la Chine (1964-2007). Tesis Doctoral. Montpellier: Université Paul Valéry-Montpellier III, 2014, p. 83, en: https://tel.archives-ouvertes.fr/tel-01077902/file/ QIBIN_2014_arch.pdf

21. Godement, François y Serra, Régine: «French Policy towards China: a Redefinition». En: Santos-Neves, Miguel y Bridges, Brian (eds.): Europe, China and the two Sars: towards a New Era. Londres: Macmillan Press, 2000, pp. 5-6; CoRnet, Christine: "The Bumpy end of the French Concession and French Influence in Shanghai». En: Rottmann, Allison, Henriot, Christian y YeH, Wen-Hsin (eds.): In the Shadow of the Rising Sun: Shanghai Under Japanese Occupation. Cambridge: Cambridge University Press, 2004, pp. 257-259. 


\section{MANUEL DE JESÚS ROCHA PINO \\ EL DESARROLLO DE LA RELACIÓN CHINA-FRANCIA: DEL RECONOCIMIENTO MUTUO 277 A LA PROYECCIÓN DE LA INICIATIVA CINTURÓN Y RUTA DE LA SEDA}

condición necesaria que Francia hiciera explícito su respeto al principio de una sola China, además de la ruptura con el régimen de Chiang Kai-shek (un antiguo aliado de Francia durante la Guerra) ${ }^{22}$. En febrero de 1964 sobrevino la ruptura de relaciones de Taipéi con París, aunque la cuestión de Taiwán se manifestó de manera recurrente en la relación sino-francesa en los años siguientes, en los casos en que París se interesaba en continuar con sus relaciones económicas con la isla y, especialmente, debido a la venta de armas de Francia a Taiwán.

Al efectuar una demostración de la independencia de su política exterior, desde el establecimiento de las relaciones sino-francesas, el gobierno francés también mantuvo la prioridad por fomentar una relación cercana y "privilegiada" con China $^{23}$. Con el tiempo, esta proximidad en la relación sino-francesa provocó que los siguientes gobiernos franceses visualizaran como otra prioridad la transmisión de sus preferencias en la relación con China hacia el proyecto de integración europeo. El periodo de gobierno gaullista definió algunas de las características estructurales de la relación sino-francesa que se mantendrían con el tiempo, incluida la política europea de Francia hacia Pekín. Algunas de estas características estructurales en el contexto de la CEE/un han sido:

1) Profundizar esta cercanía en comparación al conjunto de los otros miembros de la ceE (la mayoría de los cuales establecieron relaciones con China hasta la década de 1970); 2) conseguir una integración entre las políticas que guían el interés nacional francés hacia China con la influencia que París puede ejercer sobre la instrumentación de las políticas del proyecto de integración europeo hacia Pekín; 3) conseguir una visibilidad de las políticas francesas hacia China de forma que puedan servir como "orientación" para otros miembros de la CEE/UE ${ }^{24}$. Sin embargo, el enfoque anterior, como rasgo estructural de la relación sino-francesa, y su influencia hacia el proyecto de integración, ha generado críticas por parte de otros miembros de la CEE/UE: Francia "ha sido acusada por otras capitales europeas por usar a Europa como una plataforma enfocada en promover sus propios intereses diplomáticos y perpetuar la ilusión de que sigue siendo una gran potencia» 25 .

\subsection{La relación sino-francesa entre la Guerra Fría y la transición hacia la Posguerra Fría (1965-1995)}

Como mencionan François Godement y Régine Serra, comenzando con el establecimiento de las relaciones diplomáticas, la consideración del gobierno

22. LÜTHI, Lorenz, op. cit., pp. 127-129; Godement, François y Serra, Régine, op. cit., p. 6.

23. Dubois De Prisque, Emmanuel: "Les relations franco-chinoises, cinquante-cinq ans de faux semblants", Monde Chinois, 59(3), 2019, pp. 9-10.

24. Cabestan, Jean-Pierre: "China and European Security and Economic Interests: A French Perspective». En: Ross, Robert, Tunsjø, Øystein y Zhang, Tuosheng (ed.): us-China-Eu Relations: Managing the New World Order. Londres: Routledge, 2010, p. 123.

25. Idem. 
francés hacia China se ha derivado, por lo general, a partir de la propia percepción de su poder Ejecutivo: de esta manera, como se comentó, para el General de Gaulle las relaciones con el país asiático representaron un ejercicio de independencia de su política exterior en un momento en que Francia atravesaba el proceso de descolonizar a sus posesiones en Asia y África, al mismo tiempo que la Guerra Fría se dirimía mediante los intereses de las potencias hegemónicas en los dos bloques ${ }^{26}$.

Al mismo tiempo, hacia mediados de la década de 1960, el liderazgo de Mao en China comenzó a ser valorado con aprobación por un sector de la sociedad francesa: es conocida la visita privada del Ministro de Cultura francés, André Malraux a China, en 1965, la cual representó una profundización en las relaciones bilaterales, mientras Malraux mostraba su admiración por el proceso revolucionario y llamaba a Mao "un de Gaulle asiático» ${ }^{27}$. Desde una cercanía de posturas, ambos países condenaron la invasión soviética a Checoslovaquia en 1968.

Durante los periodos presidenciales de George Pompidou (1969-1974) y de Valery Giscard d'Estaing (1974-1981), los gobiernos franceses se orientaron a continuar con el diálogo de Estado a Estado con Pekín, mientras fomentaban las relaciones económicas, aunque de una manera menos privilegiada: con el inicio del Triángulo Estratégico en 1971, la República Popular obtuvo el reconocimiento como la única China con representación en la onU y fue iniciada la apertura de la mayoría de los países occidentales para reconocer a Pekín, su principio de «una sola China», e iniciar relaciones diplomáticas ${ }^{28}$. Un caso especial fue el de Alemania Occidental, pues no obstante lo reciente de sus vínculos oficiales (Bonn y Pekín intercambiaron embajadores en 1972), en pocos años la economía alemana se convirtió en el principal socio comercial de China en la CEE superando a Francia y el Reino Unido ${ }^{29}$.

Con el inicio de la Presidencia de François Mitterrand (1981-1995), miembro del Partido Socialista, el gobierno chino se formó algunas expectativas que probablemente resultaron frustradas: la única visita de Mitterrand a Pekín ocurrió en 1983, con el principal objetivo de presentarse con una delegación comercial. El proceso de reforma y apertura económica en China había iniciado en 1978 y, desde la perspectiva del liderazgo de Deng Xiaoping, Francia continuaba ajustándose al criterio del Segundo Mundo de la Teoría de los Tres Mundos de Mao: con el matiz de que, durante el proceso de modernización de Deng, la prioridad para China eran los intercambios en comercio y las inversiones, así como la cooperación en educación, ciencia y tecnología con el exterior ${ }^{30}$. Durante la reforma de Deng, una nueva interpretación de la Teoría de los Tres Mundos reformuló su concepto de

26. Godement, François y Serra, Régine, op. cit., pp. 6-7.

27. LIU, Kaixuan, op. cit., pp. 18-19.

28. Godement, François y Serra, Régine, op. cit., p. 7.

29. Kundnani, Hans y Parello-Plesner, Jonas: "China and Germany: Why the Emerging Special Relationship matters for Europe», Policy Brief, 55. Londres: European Council for Foreign Relations, 2012, p. 2

30. Godement, François y Serra, Régine, op. cit., p. 8. 


\section{MANUEL DE JESÚS ROCHA PINO \\ EL DESARROLLO DE LA RELACIÓN CHINA-FRANCIA: DEL RECONOCIMIENTO MUTUO 270 A LA PROYECCIÓN DE LA INICIATIVA CINTURÓN Y RUTA DE LA SEDA}

"multipolaridad" (duojibua); durante la década de 1980, el concepto de multipolaridad (o mundo multipolar) se relacionó con una redistribución del poder mundial producida por el ascenso de países ubicados en el Segundo y el Tercer Mundo (especialmente Japón, Europa Occidental y China): el principal criterio para la construcción de un mundo multipolar era la competencia por el desarrollo económico y la innovación en ciencia y tecnología "[...] acelerando el camino hacia una multipolaridad» ${ }^{31}$. El liderazgo chino evidenció sus preferencias hacia la cooperación económica con Francia, y en áreas técnicas como las telecomunicaciones y la informática, durante las visitas a París del Primer Ministro, Zhao Ziyang, en 1984 y el Secretario General del Pcch, Hu Yaobang, en 198632. La primera presencia del Jefe de Estado chino en Francia ocurrió en ocasión de la visita oficial del Presidente de la República Popular, Li Xiannian, en 1987 (en la misma acordó inversiones de capital francés en China por 747 millones de francos) $)^{33}$.

Mientras, en el ámbito regional en Asia, el gobierno francés mostraba un claro interés en mantener relaciones de cooperación y ayuda para el desarrollo con la República Socialista de Vietnam: durante la visita de Mitterrand a China, la relación franco-vietnamita se convirtió en un "tema delicado" de la agenda (si bien París había condenado la ofensiva militar vietnamita en Camboya iniciada en 1978), al mismo tiempo que el gobierno francés hacía referencia a las condiciones de una presa política china, la artista Li Shuang ${ }^{34}$. Cabe recordar que China había realizado una intervención militar punitiva en contra de Vietnam en 1979 por la incursión de tropas vietnamitas en Camboya, al mismo tiempo que Hanoi era el principal aliado soviético en la región 35 .

En 1993, Mitterrand hizo una visita oficial a Hanoi en donde reafirmó el compromiso francés para continuar con la cooperación para el desarrollo con Vietnam: la preferencia de París por cultivar los vínculos franco-vietnamitas, durante la década de 1980, osciló entre el interés por contar con un interlocutor que había compartido un pasado común con Francia, un socio estratégico para proyectar sus intereses y su poder blando en Asia, incluidas la cultura y la lengua francesas (la Francophonie), y también por cierta preocupación "paternalista» hacia una antigua colonia destruida por la guerra y en ese momento todavía aislada entre sus vecinos del Sureste de Asia, además de China, por la intervención en Camboya ${ }^{36}$. Sin

31. Garret, Banning y Glaser, Bonnie: "Chinese Assesments of Global Trends and the Emerging Era in International Relations", Asian Survey, 29(4), 1989, pp. 352-353.

32. Hou, Qibin, op. cit., p. 219.

33. Idem.

34. UPI: "French President Francois Mitterrand met Communist Party leader Hu Yaobang», UPI Archives, Pekín, 3 de mayo de 1983, en: https://www.upi.com/Archives/1983/05/03/ French-President-Francois-Mitterrand-met-Communist-Party-leader-Hu/7452420782400/

35. Liu, Kaixuan, op. cit., p. 23.

36. Wong, Reuben: Europeanization and French Policy in Asia. Tesis Doctoral. Londres: London School of Economics and Political Science, 2003, pp. 223-225, en: http://etheses.lse.ac.uk/2804/1/ U615753.pdf 
EL DESARROLLO DE LA RELACIÓN CHINA-FRANCIA: DEL RECONOCIMIENTO MUTUO A LA PROYECCIÓN DE LA INICIATIVA CINTURÓN Y RUTA DE LA SEDA

embargo, las diferentes opiniones sobre asuntos geopolíticos no fueron suficientes para provocar tensiones significativas entre el gobierno de Mitterrand y Pekín: el peligro de una ruptura (temporal) en la relación sino-francesa se presentó debido a la crisis de política interna en China al final de esta década.

Algunos meses antes del final de la Guerra Fría, sucedió la mayor crisis en la relación sino-francesa desde el establecimiento de sus relaciones debido a la represión en la Plaza de Tiananmen el 4 de junio de 1989. Frente a la represión militar en contra de la población, París asumió un liderazgo claro en la ceE para la expresión de las condenas políticas por las graves violaciones a los derechos humanos y en la imposición de sanciones económicas en contra de China: con esto, el gobierno de Mitterrand reformuló sus prioridades y se inclinó hacia la defensa de los derechos humanos ${ }^{37}$. Algunas de las sanciones impuestas por Europa fueron iniciativa del gobierno francés: el embargo a la venta de armas europeas (que continúa vigente hasta la actualidad); la suspensión de los contactos de alto nivel y de la ayuda financiera a China; la reducción de la cooperación cultural, científica y tecnológica y el otorgamiento de visas a los disidentes chinos que estuvieran en peligro $^{38}$.

Con las sanciones, la agenda normativa (relacionada con los temas de la buena gobernanza) irrumpió en la relación bilateral sino-francesa como no había ocurrido anteriormente. Sin embargo, la duración de las sanciones fue limitada por los intereses económicos y la necesidad de reanudar las relaciones de cooperación tecnológica con China ${ }^{39}$. Finalmente, con el argumento de que las «Sanciones no parecían ser efectivas» para promover la salvaguarda de los derechos humanos, Francia inició los acercamientos con Pekín para la normalización de las relaciones a partir de diciembre de 1989 y hacia 1992 la mayoría de las sanciones fueron levantadas por los miembros de la ahora Unión Europea, con la excepción del embargo de armas (en lo que contribuyó también el compromiso del liderazgo de Deng Xiaoping con las reformas económicas, mostrado durante su Viaje al Sur de 1992) ${ }^{40}$. Sin embargo, la estabilidad en los diálogos y una agenda de cooperación en la relación sino-francesa solo pudo normalizarse hasta el final del gobierno de Mitterrand: en los siguientes años, el gobierno francés se enfocó en las relaciones comerciales, y todavía un caso de venta de

37. Wellons, Patricia: «Sino-French Relations: Historical Alliance vs. Economic Reality", The Pacific Review, 7(3), 1994, p. 342.

38. Idem.; CONSEJO EuROPEO. "Declaration on China". En: Presidency Conclusions European Council. Madrid, 26 al 27 de junio de 1989, p. 25, en: https://www.consilium.europa.eu/media/20589/1989_ june_-_madrid_eng_.pdf

39. Nesshöver, Christoph: «Bonn et Paris face à Pékin (1989-1997): vers une stratégie commune?», Politique Étrangère, 64(1), 1999, p. 93.

40. Wellons, Patricia, op. cit., pp. 343-344 


\section{MANUEL DE JESÚS ROCHA PINO \\ EL DESARROLLO DE LA RELACIÓN CHINA-FRANCIA: DEL RECONOCIMIENTO MUTUO 281 A LA PROYECCIÓN DE LA INICIATIVA CINTURÓN Y RUTA DE LA SEDA}

armas de empresas francesas a Taiwán entre 1992-1993 (frustrada por la oposición de Pekín) provocó un nuevo escenario de disputas ${ }^{41}$.

En enero de 1994 fue anunciada la negativa francesa para completar la venta de armas a Taiwán, lo que contribuyó a una disminución de las tensiones ${ }^{42}$. Además, mediante un Comunicado Conjunto emitido en Pekín (con el respaldo del Primer Ministro francés Edouard Balladur y el Primer Ministro chino Li Peng), París reafirmó su reconocimiento al "gobierno de la República Popular China como el único gobierno legal de China, así como de Taiwán como parte integral del territorio chino" ${ }^{43}$. El Comunicado de 1994 también enfatizó las nuevas prioridades estructurales orientadas hacia la cooperación económica en términos pragmáticos:

Los gobiernos francés y chino tienen la intención de fortalecer sus consultas políticas, celebrar reuniones periódicas y desarrollar la cooperación económica y comercial entre los dos países. La parte china declara que las empresas francesas serán bienvenidas a ingresar en el mercado chino para competir en igualdad de condiciones ${ }^{44}$.

En abril de 1994, el Primer Ministro Balladur realizó una visita a Pekín para confirmar el enfoque económico de la relación; ${ }^{45}$ de acuerdo con Christoph Nesshöver, esta visita sirvió para corroborar que Francia "renunciaba» a hacer señalamientos sobre la situación de los derechos humanos en China: «la visita oficial de 1994 define la voluntad absoluta de no permitir que las diferencias políticas dañen el desarrollo de las relaciones económicas, mediante un verdadero modelo de "diplomacia silenciosa" "46. Posteriormente, en septiembre, el Presidente Jiang Zemin realizó una visita a París (en ocasión de la conmemoración del 30 aniversario del establecimiento de relaciones diplomáticas), con la finalidad de encauzar las relaciones bilaterales hacia el nuevo enfoque económico ${ }^{47}$.

41. Ibidem. p. 345; Godement, François: «Une politique française pour l'Asie-Pacifique?», Politique Étrangère, 60(4), 1995, p. 965.

42. Weske, Simone: "The Role of France and Germany in EU-China Relations", CAP Working Paper. Múnich: University of Munich, 2007, p. 7, en: http://www.cap.lmu.de/download/2007/2007_euchina_weske.pdf

43. Gobierno DE LA REPÚBlica FRANCESA: Communiqué conjoint franco-chinois, en date du 12 janvier 1994, sur le rétablissement de relations de coopération entre la France et la Chine. Pekín, 12 de enero de 1994, en: https://www.vie-publique.fr/discours/133004communique-conjoint-franco-chinois-en-date-du-12-janvier-1994-sur-le-r

44. Idem.

45. Le Figaro: «1994, Balladur paie le prix d'un succès économique», Le Figaro, Pekín, 10 de abril de 1994, en: https://www.lefigaro.fr/mon-figaro/2014/01/06/10001-20140106ARTFIG00432-1994-balladurpaie-le-prix-d-un-succes-economique.php

46. Nesshöver, Christoph, op. cit. p. 98.

47. Universalis: "Chine-France. Visite officielle du président Jiang Zemin en France», Universalis.fr., París, 8-14 de septiembre de 1994, en: https://www.universalis.fr/ evenement/8-12-septembre-1994-visite-officielle-du-president-jiang-zemin-en-france/ 


\section{$282 \quad$ MANUEL DE JESÚS ROCHA PINO \\ EL DESARROLLO DE LA RELACIÓN CHINA-FRANCIA: DEL RECONOCIMIENTO MUTUO A LA PROYECCIÓN DE LA INICIATIVA CINTURÓN Y RUTA DE LA SEDA}

De acuerdo con Jean-Vincent Brisset, los términos del Comunicado de 1994 «Sacrificaron los principios mientras ignoraron los intereses, con este cambio los líderes franceses de la época perdieron toda consideración de sus homólogos

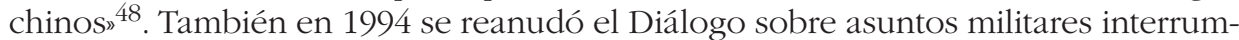
pido en 1989, aunque las relaciones de cooperación mostraron un claro aumento de actividad a partir de 1995: con el inicio del periodo presidencial de Jacques Chirac se instrumentaron de forma plena los nuevos principios pragmáticos de la relación ${ }^{49}$.

El gobierno de Mitterrand se ocupó de instrumentar una agenda en la relación sino-francesa que en ocasiones fue percibida por Pekín como correspondiente a coyunturas que mantenían una prioridad para Francia, pero que no se correspondían con el interés chino y el contenido de la agenda bilateral: en casos como las sanciones económicas y la venta de armas a Taiwán, considerados por Pekín como intervencionistas, e incluso por la preferencia por privilegiar a las relaciones de cooperación con Vietnam. Posteriormente, durante una visita del Presidente Chirac a China, el Primer Ministro Li Peng le comentó que «Mitterrand había cerrado la puerta abierta por de Gaulle» (cabe recordar que Li Peng fue uno de los líderes chinos señalados como los organizadores de la represión en 1989) $)^{50}$.

Sin embargo, con el deterioro de la relación manifestado al final del gobierno de Mitterrand, el gobierno francés decidió reestructurar sus políticas de aproximación hacia China teniendo como prioridad, a partir de 1994, a las relaciones económicas: en dicha acción fue importante la consideración de que otros miembros de la uE, especialmente Alemania (en 1993 y 1995 el Canciller Helmut Kohl había realizado visitas a China con claros resultados económicos), estrechaban sus relaciones económico-comerciales y de inversión con China, mientras la relación sinofrancesa se mantenía ocupada (y rezagada) en las tensiones normativas e incluso estratégicas (como en el caso de la venta de armas a Taiwán, con una consideración de lucro a corto plazo $)^{51}$. La reestructuración de la relación sino-francesa fue instrumentada por el gobierno presidido por el gaullista Chirac.

48. BRISSET, Jean-Vincent: "Les relations franco-chinoises: entre normalité et brouille», La revue internationale et stratégique, 77, 2010, p. 134

49. Godement, François y Serra, Régine, op. cit., p. 17.

50. Ibidem. p. 9; LA VAnguardia: "Fallece Li Peng, represor de Tiananmen", La Vanguardia, 23 de julio de 2019, en: https://www.lavanguardia.com/internacional/20190723/463672316334/li-pengrepresor-tiananmen-fallece.html

51. Nesshöver, Christoph, op. cit. pp. 97-98; Tzogopoulos, George: «A Friend of China, Helmut Kohl, passes away», China.org.cn, 19 de junio de 2017, en: http://www.china.org.cn/opinion/2017-06/19/ content_41056323.htm 
MANUEL DE JESÚS ROCHA PINO

EL DESARROLLO DE LA RELACIÓN CHINA-FRANCIA: DEL RECONOCIMIENTO MUTUO 283 A LA PROYECCIÓN DE LA INICIATIVA CINTURÓN Y RUTA DE LA SEDA

3. La RELACiÓn SinO-FRANCESA DURANTE la PRESIDENCIA DE JACQUES ChIRAC (1995-2007).

LA AGENDA DE ASOCIACIÓN ESTRATÉGICA DE 1997

Desde el inicio de las relaciones diplomáticas en 1964, se ha podido constatar una coordinación entre algunos aspectos de la agenda de la política exterior de Francia con la de China, teniendo como principal consideración a los intereses nacionales de los actores, por lo general en asuntos puntuales y dependiendo de la prioridad que los gobiernos franceses habían otorgado a las cuestiones normativas. A partir del inicio de la presidencia de Jacques Chirac, además de la agenda bilateral, se presentó uno de los periodos más claros en los que el gobierno francés ejerció un liderazgo sobre las políticas de aproximación de la uE hacia China: en un primer caso relacionado con el ámbito normativo y multilateral que culminó con la instauración del Diálogo China-ue sobre Derechos Humanos.

Este caso fue el apoyo francés para que China dejara de estar sujeta a las evaluaciones anuales realizadas por la entonces Comisión de Derechos Humanos de la ONU (CDH) a partir de 1995 (cuyas investigaciones eran realizadas desde 1989, como consecuencia de la represión en Tiananmen, e impulsadas por Estados Unidos y otros países europeos como Dinamarca) ${ }^{52}$ : hacia 1995, Pekín comenzó a mostrar su molestia debido a que «el periodo de sanciones había durado mucho tiempo" y los cuestionamientos en la ONU eran considerados como una forma de "desprecio» ${ }^{53}$. Durante el primer semestre de aquel año, la Presidencia del Consejo Europeo correspondió a Francia: como señala René Dorient, el gobierno de Chirac "propuso, por tanto, un método prefiriendo el diálogo a puerta cerrada a las condenas públicas. En el corazón de esta política figuró el abandono del apoyo a las resoluciones sobre la situación de los derechos humanos en China de la $\mathrm{CDH}{ }^{54}$. Hacia finales de 1995, durante la Cumbre del Consejo Europeo, realizada en Madrid, la Comisión Europea emitió su primer Documento Estratégico sobre China titulado Una política a largo plazo para las relaciones China-UE. En el Documento fue definido el nuevo mecanismo de diálogo sino-europeo en la materia:

Un nuevo diálogo especialmente enfocado en los derechos humanos ha sido inaugurado recientemente a sugerencia de China. Este diálogo se centra en una reunión semestral entre la Troika de la uE y el gobierno chino, que no solo mejorará

52. Dorient, René: «Un septennat de politique asiatique: quel bilan pour la France?», Politique Étrangère, 67(1), 2002, p. 176; TAYLOR, Max Roger: «Inside the Eu-China Human Rights Dialogue: assessing the Practical delivery of the Eu's Normative Power in a Hostile Environment», Journal of European Integration, diciembre, 2020, pp. 4-5.

53. Kirchner, Emil J. y Christiansen, Thomas: "The Political Dialogue of eu-China Relations», ponencia presentada en la 43 uAces Conference, Leeds, 2013, p. 7, en: https://www.uaces.org/documents/papers/1301/kirchner-christiansen.pdf

54. Dorient, René, op. cit. p. 176. 
el flujo de información, sino que también brinda la oportunidad para una discusión detallada que conduzca a acciones concretas ${ }^{55}$.

De acuerdo con el proceso anterior, las presiones del gobierno chino provocaron que Francia (y también Alemania) establecieran un consenso con China en un tema de la buena gobernanza, como los derechos humanos, que además sirvió para incentivar las relaciones de cooperación, especialmente en el diálogo político y las relaciones económicas entre París y Pekín: posteriormente, en marzo de 1996, durante la Primera Cumbre del Encuentro Asia-Europa (Asia-Europe Meeting, ASEM, por sus siglas en inglés), en Bangkok, Li Peng sostuvo reuniones con Chirac y Kohl con la finalidad de acordar "la suspensión de las resoluciones de la CDH de la ONU en las que se criticaba su situación en China ${ }^{56}$. Durante el gobierno de Chirac, París encabezó la coordinación de un "diálogo constructivo" sino-europeo sobre el tema y, en abril de 1997, Francia y Alemania se negaron a presentar un proyecto de resolución crítica hacia China en la $\mathrm{CDH}^{57}$. Igualmente, las acciones del gobierno francés tenían una finalidad instrumental al "promover un impacto positivo en los contratos de China para el empresariado francés " 58 .

El Diálogo China-ue sobre Derechos Humanos, iniciado en 1995, desde un principio fue coordinado por la Comisión Europea y el Consejo Europeo, y sin una participación del Parlamento Europeo (integrado por parlamentarios con tendencias más críticas hacia China y con la potestad de aplicar políticas vinculantes) $)^{59}$. El Diálogo se ha mantenido de manera casi ininterrumpida desde 1997 y por lo general se estructura como un encuentro de carácter político y deliberativo sin presentar posiciones que la parte china pudiera considerar como intervencionistas en sus asuntos internos ${ }^{60}$. Sin embargo, de una manera crítica, John Fox y François Godement definen a estas acciones como una política de "compromiso incondicional» de la UE hacia China: «[...] una política que otorga a China el acceso a todo lo económico así como a otros tipos de beneficios con Europa sin requerir

55. COMisión EuRopea: A Long-term Policy for China-Europe Relations, Bruselas, com (1995) 279/ final, p. 7, 1995, en: https://eeas.europa.eu/archives/docs/china/docs/com95_279_en.pdf; ver: BAKER, Phillip: "Human Rights, Europe and the People's Republic of China", The China Quarterly, 169, 2002, pp. 58-60.

56. Rocha Pino, Manuel: «La simetría en la asociación estratégica República Popular China-Unión Europea", Estudios de Asia y África, 45(1), 2010, pp. 131-133; Wong, Reuben: The Europeanization of French Foreign Policy: France and the Eu in East Asia. Nueva York: Palgrave Macmillan, 2006, pp. 94-95.

57. Idem.

58. Lequesne, Christian: "La politique étrangère de Jacques Chirac ou la France sans surprise», DGAP-Analyse Frankreich, 2. Berlin: Forschungsinstitut der Deutschen Gesellschaft für Auswärtige Politik, 2007, p. 10, en: https://www.ssoar.info/ssoar/bitstream/handle/document/13137/ssoar-2007lequesnela_politique_etrangere_de_jacques.pdf?sequence=1

59. Kinzelbach, K. y Thelle, Hatla: "Talking Human Rights to China: An Assessment of the Eu's Approach", The China Quarterly, 205, 2011, pp. 61-62.

60. Ibidem. p. 67. 


\section{MANUEL DE JESÚS ROCHA PINO \\ EL DESARROLLO DE LA RELACIÓN CHINA-FRANCIA: DEL RECONOCIMIENTO MUTUO 285 A LA PROYECCIÓN DE LA INICIATIVA CINTURÓN Y RUTA DE LA SEDA}

nada a cambio ${ }^{61}$. Si bien en el caso de la relación sino-francesa, la disminución de las tensiones alrededor de los temas de la buena gobernanza contribuyó a la conformación de la asociación estratégica China-Francia de 1997.

Un resultado de la aproximación sino-francesa entre 1995-1997 fue la negociación para conformar su acuerdo de asociación estratégica a partir de 1997, además de un conjunto de declaraciones diplomáticas que buscaron aproximar las posturas de ambos actores en asuntos de la actualidad internacional de ese momento, en una dinámica de diálogo y acomodación de intereses que se extendió durante el resto del gobierno de Chirac hasta 2007, y en el que sobresalió el diálogo sobre el mundo multipolar (que fue particularmente relevante durante la coyuntura de la crisis internacional de 2003) ${ }^{62}$. El establecimiento de la asociación estratégica sino-francesa se correspondía igualmente con una mayor actividad de la política exterior francesa en Asia: además de China, Francia había establecido una asociación estratégica con Japón en 1996 y lo haría con India en $1998^{63}$. Algunos de los temas de la agenda sino-francesa fueron:

1) La asociación estratégica China-Francia de 1997 y el diálogo sobre la multipolaridad: el gobierno francés inició un diálogo alrededor del concepto chino sobre la "construcción de un mundo multipolar" al mismo tiempo que se incrementaban los diálogos de alto nivel y las visitas oficiales recíprocas ${ }^{64}$. El diálogo sobre la multipolaridad inició un proceso paulatino de acomodación a una mayor presencia china en el sistema internacional y de una manera anticipada a las acciones realizadas posteriormente por otros miembros de la uE (como Alemania, España y el Reino Unido quienes establecieron acuerdos de asociación estratégica con China hasta la década del 2000).

De esta forma, en 1997, Francia se convirtió en el primer país de la uE en establecer una "asociación estratégica" con China (el antecedente inmediato en Europa sobre este tipo de acuerdos con Pekín era la asociación estratégica China-Rusia de 1996, la cual había sido una respuesta, a su vez, hacia los planes de ampliación de la OTAN en los países de Europa del Este) ${ }^{65}$. En la Declaración Conjunta sobre la asociación estratégica sino-francesa se señala:

China y Francia, como miembros permanentes del Consejo de Seguridad de la ONU, comparten una responsabilidad especial en los asuntos internacionales, en

61. Fox, John y Godement, François: A Power Audit of Eu-China Relations. Londres: European Council of Foreign Relations, 2009, p. 2, en: https://www.ecfr.eu/page/-/ECFR12_-_A_POWER_AUDIT_OF_ EU-CHINA_RELATIONS.pdf

62. WOnG, Reuben: "China's Rise: Making Sense of Eu Responses», Journal of Contemporary East Asia Studies, 2(2), 2013, p. 120.

63. DORIEnT, René, op. cit. p. 175.

64. Lequesne, Christian, op. cit. p. 9; Mengin, Françoise: «La politique chinoise de la France. Du mythe de la relation privilégiée au syndrome de la normalisation", Critique internationale, 110, 2001, p. 104.

65. VÉDRINE, Hubert: "L'Émergence de la Chine vue de France», Politique Étrangère, 3, 2006, pp. 484-485. 
los que una China que aumenta en fortaleza y una Europa unida jugarán un papel importante como parte del nuevo mundo multipolar. Profundizar las relaciones sino-francesas y las relaciones asiático-europeas ejercerá un impacto positivo en los desarrollos globales. Los dos países decidieron intensificar la cooperación y acelerar el proceso de mover el mundo en la dirección multipolar ${ }^{66}$.

Los temas prioritarios en la asociación estratégica se orientaron a fortalecer la multipolaridad, contribuir a la reforma de Naciones Unidas, promover el desarme, la protección del medio ambiente, la lucha contra el crimen y el terrorismo, fortalecer la ayuda al desarrollo, el apoyo al comercio multilateral, etc. ${ }^{67}$. El acercamiento discursivo contribuyó al posterior entendimiento entre el gobierno de Chirac y el liderazgo en Pekín encabezado por el Secretario General del Pcch, Hu Jintao, a partir de 2002 (Hu fue elegido Presidente de China en 2003).

Como parte de la agenda de la asociación estratégica, Francia adoptó el concepto del mundo multipolar debido a la promoción de sus intereses nacionales, en tanto que estos se habían mantenido como una característica estructural en la relación sino-francesa, al mismo tiempo que el concepto de multipolaridad proporcionaba un puente en el diálogo de la relación uE-China (aunque el gobierno francés no tomaba en cuenta la tensión que podría existir entre el discurso del mundo multipolar y la preferencia europea por el multilateralismo) ${ }^{68}$. Como menciona Reuben Wong, sobre la política exterior francesa del gobierno de Chirac: «todavía conservó una reliquia gaullista al aspirar a una grandiosa "diplomacia declaratoria", como en el caso de su discurso sobre la multipolaridad y la actitud anti-hegemonista hacia EEUU presente en sus relaciones con China" 69 .

En un inicio, la asociación estratégica sino-francesa estructuró una coordinación de políticas por medio del acercamiento de los discursos diplomáticos: de esta manera hubo una aproximación más clara entre los intereses compartidos en la relación bilateral (a través de un proceso de acomodación) ahora orientados hacia la cooperación económica y la participación en organismos internacionales. Sin embargo, el proceso de aproximación de discursos e intereses mantuvo como límite el compromiso francés con la alianza transatlántica y el respeto al statu quo estadounidense: la presidencia francesa «esperaba ver el surgimiento de un mundo multipolar, aunque esto no significaba que quisiera oponerse a Estados Unidos $[\ldots]_{3} 70$.

66. Ministerio De Asuntos Exteriores De China: China and France aim to build a Longterm full Partnership, Pekín, 16 de mayo de 1997, en: https://www.fmprc.gov.cn/mfa_eng/zil iao_665539/3602_665543/3604_665547/t18031.shtml

67. Mengin, Françoise, op. cit. p. 104

68. Jørgensen, Knud Erik y Wong, Reuben: «Social Constructivist Perspectives on China-Eu Relations». En: WANG, Jianwei y SONG, Weiqing (eds.): China, the European Union, and the International Politics of Global Governance. Nueva York: Palgrave-Macmillan, 2016, p. 61.

69. WONG, Reuben, 2003, op. cit. p. 266.

70. Zhang, Zuqian: "La politique étrangère de la France entre continuité et ajustements postguerre froide», La revue internationale et stratégique, 45, 2002, p. 119. 
MANUEL DE JESÚS ROCHA PINO

EL DESARROLLO DE LA RELACIÓN CHINA-FRANCIA: DEL RECONOCIMIENTO MUTUO 287 A LA PROYECCIÓN DE LA INICIATIVA CINTURÓN Y RUTA DE LA SEDA

2) El discurso sobre la construcción de un mundo multipolar y la creación del Encuentro Asia Europa: la Declaración Conjunta de la asociación estratégica sinofrancesa de 1997 hace referencia a las relaciones euro-asiáticas, institucionalizadas previamente, en 1996, mediante el diálogo mantenido entre la uE y la Asociaciones de Naciones del Sureste de Asia (ANSEA) además de China, Corea del Sur y Japón (el futuro grupo ANSEA + 3) para la conformación del ASEM. Los antecedentes del mecanismo se remiten a una propuesta realizada por el entonces Primer Ministro de Singapur, Goh Chok Tong, durante una visita oficial a París en 1994, al entonces Primer Ministro Balladur para convocar al establecimiento de un diálogo entre Asia y Europa (posteriormente la propuesta fue presentada por Goh durante una intervención en el Foro Mundial de Davos de 1995) ${ }^{71}$ : la iniciativa para cimentar un mecanismo de diálogo interregional euro-asiático fue retomada con el inicio de la presidencia de Chirac.

El gobierno de Singapur tuvo algunas razones específicas para invitar en primera instancia a Francia para transformar las percepciones francesas sobre Asia en "una cumbre tangible e institucionalizada entre Europa y Asia» ${ }^{72}$ : a) previamente, EEUu había rechazado la petición de la uE para obtener la condición de observadora en el Foro de Cooperación de Asia Pacífico (APEC) en una situación que inconformó especialmente al gobierno francés; b) se consideró que Francia tenía una orientación estratégica a largo plazo y, por tanto, podría ser más receptiva para entablar un diálogo interregional; c) Singapur esperaba beneficiarse directamente atrayendo más inversiones francesas ${ }^{73}$.

En la construcción del ASEM fueron igualmente relevantes la transmisión de los objetivos de la política exterior alemana hacia Asia (el denominado Asienkonzept de 1993) para las políticas de la uE, como el liderazgo diplomático del gobierno de Chirac y el apoyo del Consejo Europeo (que en ese momento iniciaba la instrumentación de la política específica de la uE hacia Asia con la New Asia Strategy -NAS- de 1994) ${ }^{74}$. Mediante la agenda del ASEm los países europeos, y particularmente Francia, buscaban dos objetivos básicos: 1) incrementar las relaciones comerciales y de cooperación con Asia del Este (en un momento en que la región asiática se definía como una de las regiones con mayores posibilidades de desarrollo), en lo que resultaban prioritarios los acercamientos de la uE hacia China. 2) La conformación de un diálogo entre Europa y Asia inexistente hasta ese momento (mientras que en la relación transpacífica existía el APEC y en la relación Estados

71. Ayrault, Jean-Marc: The Asia-Europe Meeting on its 20th Anniversary. Singapur: Asia-Europe Foundation, 2016, en: https://www.asef.org/press/corporate/news-3894-theasia-europe-meeting-on-its-20th-anniversary

72. European Background Study: ASEM in its Tenth Year, Looking Back, Looking Forward. An evaluation of ASEM in its first decade and an exploration of its future possibilities. Helsinki: University of Helsinki Network for European Studies, 2006, en https://www.mofa.go.jp/policy/economy/asem/ tenth/report3.pdf

73. Ibidem. p. 21-22.

74. Ibidem. p. $18-20$. 
EL DESARROLLO DE LA RELACIÓN CHINA-FRANCIA: DEL RECONOCIMIENTO MUTUO A LA PROYECCIÓN DE LA INICIATIVA CINTURÓN Y RUTA DE LA SEDA

Unidos-ue, la Nueva Agenda Transatlántica de 1995) ${ }^{75}$. Desde la perspectiva del diálogo político sino-francés, las actividades del ASEM se convirtieron en una manifestación de la construcción del mundo multipolar y ofrecieron un nuevo ámbito de cooperación multilateral para la agenda sino-francesa.

3) Igualmente en el ámbito multilateral, a partir de 1995-1997, Francia y Alemania promovieron la adhesión temprana de China a la Organización Mundial del Comercio (China fue aceptada en la omc en 2001) ${ }^{76}$.

4) Un caso de aproximación bilateral debido a una clara acción de equilibrio hacia un tercer país, que definió a la relación sino-francesa durante la primera década del siglo 21, ocurrió en 2003: es decir, el contexto internacional que llevó al rechazo por parte de China y Rusia (y algunos miembros de la uE como Alemania y Francia) hacia los planes de la invasión militar de EEuU a Irak. Esta coyuntura de crisis se denominó el "distanciamiento» o la "brecha» transatlántica frente a las políticas unilaterales del gobierno de Bush (sin que esto significara que Francia desistiera de sus compromisos estratégicos en la OTAN): el distanciamiento se debió en lo fundamental «a un profundo desacuerdo respecto a la naturaleza de la amenaza terrorista y a la estrategia más adecuada para enfrentarla ${ }^{77}$.

El consenso por la desaprobación hacia la invasión a Irak llevó a la relación sino-francesa al mantenimiento de un diálogo político y de cooperación en el ámbito multilateral sin precedentes: como resultado, París apoyó que aumentara el nivel de la cooperación entre la ue y China mediante el establecimiento de la asociación estratégica China-uE en octubre de 2003 e impulsó un debate en el Parlamento Europeo en favor del levantamiento del embargo de armas europeo vigente desde 1989 (igualmente con la finalidad de que la industria militar francesa pudiera acceder al mercado de armas en China $)^{78}$. El debate sobre el levantamiento del embargo abarcó entre 2003-2005 y fue abandonado por la oposición de Washington y algunos miembros de la ue como el Reino Unido y Países Bajos a la venta de armas europeas a China, sumado en noviembre de 2005 el inicio del periodo de gobierno de Angela Merkel en Alemania, entonces más cercana a las posiciones estadounidenses.

La oposición diplomática de Francia a la intervención estadounidense en Irak fue más activa que la de China. Mientras el gobierno francés amenazaba con vetar la iniciativa que buscaba la intervención en Irak en el Consejo de Seguridad de la ONu, China examinaba solo la abstención: en ese momento el discurso sobre la multipolaridad se relacionó con la estabilidad del sistema internacional y el rechazo a la

75. HängGI, Heiner: "Interregionalism as a Multifaceted Phenomenom: In Search of Typology». En: HäNGGI, Heiner, Roloff, Ralf y RüLAND, Jürgen (eds.): Interregionalism and International Relations. Londres: Routledge, 2006

76. Godement, François y Serra, Régine, op. cit., p. 25.

77. Sanahuja, Jose Antonio: «Europa y Estados Unidos después de Bush. La difícil reconstrucción del vínculo trasatlántico", Foreign Affairs Latinoamérica, 8(4), 2008, p. 100

78. Lequesne, Christian, op. cit. p. 10. 


\section{MANUEL DE JESÚS ROCHA PINO \\ EL DESARROLLO DE LA RELACIÓN CHINA-FRANCIA: DEL RECONOCIMIENTO MUTUO 289) A LA PROYECCIÓN DE LA INICIATIVA CINTURÓN Y RUTA DE LA SEDA}

guerra $^{79}$. Por otra parte, algunos analistas chinos señalaron que era improbable que sucediera una ruptura en la relación franco-estadounidense, un evento que probablemente remitía a la década de 1960:

Las demostraciones de independencia del General de Gaulle frente a Washington fueron parcialmente el fruto del carácter intransigente del General, y no la expresión de una estrategia nacional. Por lo tanto, los analistas chinos percibieron que la disputa franco-estadounidense sobre Irak se refería sólo a la forma y no a la sustancia del problema ${ }^{80}$.

De manera similar, la crisis de 2003 propició la oportunidad para que, como parte de la agenda de la asociación estratégica sino-francesa, continuara la estructuración de un discurso alrededor del concepto del mundo multipolar y la importancia de la estabilidad. Este discurso compartido estaba motivado por los intereses nacionales de Francia y su posición como potencia líder en Europa y el interés de China por contar con interlocutores que contribuyeran a una diversificación de su política exterior, como prevención en caso de que eEuu se decidiera a instrumentar políticas de contención en su contra. Como menciona Dario Battistella:

[...] en la definición de un sistema multipolar como un mundo caracterizado por la existencia de "varios polos de estabilidad", las propias autoridades francesas sitúan su conducta diplomática en la perspectiva de la teoría realista y la práctica de la Realpolitik, según las cuales el orden es sinónimo de un sistema estable ${ }^{81}$.

Como parte del proceso de profundización de la relación, en 2004 ambos actores elevaron su asociación al nivel de una asociación estratégica global (perteneriat stratégique global) ${ }^{82}$. Sin embargo, en la Declaración Conjunta de 2004 sobre la asociación estratégica global no fue incluido el concepto de multipolaridad sino el de multilateralismo: «Francia y China recuerdan su apego al multilateralismo como el medio apropiado para prevenir y resolver de manera efectiva las crisis, en respuesta a las amenazas y desafíos globales y regionales» ${ }^{83}$. Como menciona François Mengin:

[...] el orden multipolar en un sentido normativo de estabilidad es un oxímoron ya que en la perspectiva de la Realpolitik que sostiene Francia, el orden internacional

79. ZHENG, Ruolin: «Un intérêt renouvelé pour la France», La revue internationale et stratégique, 53, 2004, p. 114

80. Idem.

81. Battistella, Dario: "L'ordre international. Portée théorique et conséquences pratiques d'une notion réaliste», La revue internationale et stratégique, 54, 2004, p. 80.

82. Gobierno De la RePública FRANCESA: Déclaration Conjointe Chine France (01-2004): "Approfondir le partenariat global stratégique franco-chinois pour promouvoir un monde plus sûr, plus respectueux de sa diversité et plus solidaire». París, 9 de mayo de 2004, en: http://www.amb-chine.fr/fra/zfzj/ zzgx/t96906.htm

83. Idem 


\section{EL DESARROLLO DE LA RELACIÓN CHINA-FRANCIA: DEL RECONOCIMIENTO MUTUO A LA PROYECCIÓN DE LA INICIATIVA CINTURÓN Y RUTA DE LA SEDA}

es inconcebible sin el desequilibrio unipolar. Desear "construir un mundo multipolar» para impedir «el caos político» está envuelto en un deseo piadoso ${ }^{84}$.

El carácter coyuntural de las aproximaciones sino-francesas mantenidas durante el gobierno de Chirac se demostró con el inicio del periodo presidencial de Nicolas Sarkozy en 2007, quien agregó a la agenda los temas normativos (especialmente la situación de los derechos humanos en China) y nuevas prioridades en la relación económica. A partir de 2005, la relación económico-comercial sinoeuropea comenzó a generar una inquietud creciente en la uE debido al déficit mantenido con China: entre 2006-2007, las cuestiones económicas se convirtieron en un tema principal de la agenda, y durante la 10 Cumbre China-uE realizada en 2007, la uE logró negociar un compromiso, por parte de Pekín, para una reducción del ritmo del crecimiento del déficit en los términos de un «un comercio más balanceado y una asociación económica" 85 .

Un tema que afectaba particularmente a Francia (además de Italia y España) era la cuestión sobre la competencia de las manufacturas de textiles y de calzado, importadas desde China, por lo que París apoyó las investigaciones antidumping en la uE para restringirlas ${ }^{86}$. En octubre de 2006 , Chirac realizó un viaje oficial a China teniendo a la economía (y las oportunidades en China para empresas francesas como Airbus, Société Générale, Alstom, Carrefour, Peugeot, etc) como el tema principal de la agenda. En 2006 el déficit comercial para Francia con China había sido de 7,788 millones de euros $^{87}$.

84. Mengin, Françoise, op. cit. p. 110. De manera similar, en 2005 el discurso oficial de la UE subrayó su compromiso con el multilateralismo haciendo una distinción con el discurso chino sobre la multipolaridad; como señaló la entonces Comisaria Europea de Relaciones Exteriores y Política de Vecindad, Benita Ferrero-Waldner, en su discurso para conmemorar el 30 aniversario del establecimiento de relaciones China-UE: "Como actores globales, China y la uE están obviamente interesadas en la naturaleza de la política global en el siglo 21. Algunos han señalado sobre construir un "mundo multipolar». Para la UE, sin embargo, no es el número de polos lo que cuenta, sino la base sobre la que operan. Nuestra visión es un mundo gobernado por reglas creadas y monitoreadas por instituciones multilaterales. Y sé que China comparte este enfoque». Ver: Comisión EuropeA: The EU, China and the Quest for a Multilateral World. Conference to mark the 30th anniversary of EU-China relations: Institut Français de Relations Internationales - China Institute of International Studies SPEECH/05/414, Bruselas, 4 de julio de 2005, en: https://ec.europa.eu/commission/presscorner/detail/en/sPEECH_05_414

85. Consejo Europeo: 10th China-eu Summit Beijing, 28 November 2007 Joint Statement 16070/07 (Presse 279), Pekín, 28 de noviembre, de 2007, p. 2, en: https://www.consilium.europa.eu/ ueDocs/cms_Data/docs/pressData/en/er/97355.pdf

Entre 2005-2008 el déficit comercial de la uE con China fue (en miles de millones de euros): -109.3 en 2005; -132.1 en 2006; -162 en 2007; y -170.8 en 2008. Ver: EuROSTAT: "Record EU Deficit in Trade in Goods with China of $€ 180$ billion in 2015...", Newsrelease, 138/2016, p. 2, en: https://ec.europa.eu/ eurostat/documents/2995521/7553974/6-12072016-BP-EN.pdf/67bbb626-d55f-4032-8c24-48e4c9f78c3a

86. Comino, Anna: "A Dragon in Cheap Clothing: What Lessons can be Learned from the Eu-China Textile Dispute», European Law Journal, 13(6), 2007, pp. 829-831; wong, Reuben, 2013, op. cit. p. 117.

87. eurostat: "Les échanges commerciaux entre l'ue27 et la Chine ont augmenté de $150 \%$ entre 2000 et 2006", Communiqué de Presse, 160/2007, p. 2, en: https://ec.europa.eu/eurostat/ documents/2995521/5053834/6-26112007-AP1-Fr.PDF/c600f46f-ab02-4891-a04f-5c1cc562fc44?version=1.0 
MANUEL DE JESÚS ROCHA PINO

EL DESARROLLO DE LA RELACIÓN CHINA-FRANCIA: DEL RECONOCIMIENTO MUTUO 291 A LA PROYECCIÓN DE LA INICIATIVA CINTURÓN Y RUTA DE LA SEDA

4. La relación Sino-francesa durante la Presidencia de Nicolas Sarkozy (2007-2012).

LA CRISIS Y EL PRAGMATISMO ECONÓMICO

En 2007 tuvieron lugar elecciones presidenciales en Francia y Nicolas Sarkozy, el candidato de centro-derecha, resultó el ganador para el siguiente periodo de gobierno 2007-2012: desde su campaña electoral, Sarkozy había mostrado, mediante la reiteración de su "atlantismo", su intención por iniciar una aproximación hacia EEUU $^{88}$ : una acción anunciada durante su visita a Washington en septiembre de 2006 (como contraste, la candidata del Partido Socialista, Ségolène Royal, realizó una visita a China durante su tiempo de campaña en enero de 2007) ${ }^{89}$.

Durante el inicio del periodo de gobierno de Sarkozy, durante 2007-2009, se comenzó a producir un distanciamiento entre China y Francia, en esta ocasión en el contexto de una mayor aproximación del gobierno francés a las preferencias de la política exterior estadounidense. De la misma forma, sobresalió una actitud más crítica de París hacia la situación de la democracia y los derechos humanos en China $^{90}$. Los desacuerdos se mostraron evidentes en la relación bilateral por una posición más crítica de Francia hacia ciertos temas de la política interior china, algo que provocó la suspicacia en Pekín hacia el nuevo presidente francés.

Un objetivo de la política exterior del gobierno de Sarkozy fue el de superar al denominado "compromiso incondicional" que había determinado a las relaciones entre la UE y China (especialmente durante 2003-2005). Sin embargo, París consideró que su aproximación hacia China podría desarrollarse sin interferir en las relaciones económicas; como señaló Sarkozy durante su primera visita a Pekín en noviembre de 2007 (durante la cual consiguió contratos por 20 mil millones de euros para empresas francesas): "China tiene un papel vital que jugar [...] para no permitir que los desequilibrios se acumulen hasta el punto de que ya no sepamos solucionarlos ${ }^{91}$. En este sentido, Sarkozy propuso al liderazgo chino una relación mayormente "transaccional", en lugar de una estratégica, y que además incluyera una activa agenda normativa: "los encuentros políticos de alto nivel se utilizaron con frecuencia para facilitar el rumbo para los contratos comerciales a gran escala»

88. Coulomb-Gully, Marlène: «Le corps présidentiel. Représentation politique et incarnation dans la campagne présidentielle française de 2007", Mots. Les langages du politique, 89, 2009, p. 32.

89. De Charette, Hervé: "Nicolas Sarkozy et la politique étrangère de la France: entre changement et continuité", Revue internationale et stratégique, 70, 2008, p. 11; LE MONDE: "La "bravitude» de Ségolène Royal en Chine», Le Monde, 6 de enero de 2007, en: https://www.lemonde.fr/societe/article/2007/01/06/la-bravitude-de-segolene-royal-en-chine_852795_3224.html

90. Seaman, John y Ekman, Alice: "France and China: A not so «special» Relationship». En: HuoTARI, Mikko et. al. (eds.): Mapping Europe-China Relations. A Bottom-Up Approach. Bruselas: European Think-tank Network on China, 2015, p. 26; De ChareTte, Hervé, op. cit. p. 11.

91. Challenges: "Sarkozy en Chine: les contrats deux fois plus importants que prévu», Challenges, 27 de noviembre de 2010, en: https://www.challenges.fr/entreprise/ sarkozy-en-chine-les-contrats-deux-fois-plus-importants-que-prevu_380146 
al mismo tiempo que «se adoptaba una postura más dura hacia China y los derechos humanos» ${ }^{92}$.

Así mismo, el gobierno francés decidió modificar los términos de la «asociación estratégica global» ("perteneriat stratégique global») al ser denominada ahora como una "asociación estrecha" (parteneriat étroit»), un concepto introducido por Sarkozy en su discurso oficial en Pekín, en presencia de Hu Jintao, y que provocó extrañeza en la diplomacia China ${ }^{93}$.

De esta manera, prevalecieron una variedad de prioridades ajustadas a una agenda que resultó reactiva e incluso contradictoria. Las posiciones de la política exterior francesa pudieron ir desde el alineamiento a las políticas de EEUU hacia China, la crítica a la situación de los derechos humanos y los señalamientos sobre la situación de las protestas en el Tỉbet de 2008 y la exigencia para que China se sumara a las sanciones contra Irán, al mismo tiempo que la diplomacia económica de Sarkozy pedía una mayor apertura del mercado chino ${ }^{94}$. Debido a la represión de las protestas en Lhasa, el presidente francés amenazó con no asistir a la inauguración de los Juegos Olímpicos de Pekín, en agosto de 2008, aunque finalmente asistió: la oposición política francesa lo calificó como «incoherente» y "ganador de la medalla de oro a la hipocresía "95.

La relación sino-francesa alcanzó su mayor nivel de tensión diplomática durante los meses de noviembre y diciembre de 2008 debido al anuncio del encuentro del presidente francés con el Dalái Lama en el puerto polaco de Gdansk («en ocasión de la celebración del 25 aniversario de la concesión del premio Nobel de la Paz a Lech Walesa") ${ }^{96}$. La reunión entre ambos se realizaría el 6 de diciembre pese a las advertencias de Pekín de que podría realizar actos de boicot en contra de productos franceses. De manera coincidente, en ese momento Francia ejercía la Presidencia rotatoria del Consejo Europeo (segundo semestre de 2008) y le correspondía la organización de la 11 Cumbre China-Unión Europea en Lyon.

La sanción de China a lo que consideró una intromisión del gobierno francés en sus asuntos internos consistió en anunciar, el 26 de noviembre, a las autoridades de la uE que posponía de manera indefinida la realización de la Cumbre

92. Seaman, John y Ekman, Alice op. cit. p. 26.

93. Presidencia de la república francesa: Déclaration de M. Nicolas Sarkozy, Président de la République, sur les relations franco-chinoises, à Pékin le 26 novembre 2007, Pekín, 26 de noviembre de 2007, en: https://www.elysee.fr/nicolas-sarkozy/2007/11/26/declaration-de-m-nicolas-sarkozy-president-dela-republique-sur-les-relations-franco-chinoises-a-pekin-le-26-novembre-2007

94. Heng-Lim, Yves: "Bilan et perspectives de la politique chinoise de la France», La revue internationale et stratégique, 29, 2014, pp. 35-36; DE LA MAISONNEuvE, Eric: "Les relations franco-chinoises: brouille ou malentendu?», Actuel, 24, 2008, pp. 1-2, en: http://www.societestrategie.fr/pdf/actuel24.pdf

95. Le Parisien: "Nicolas Sarkozy est arrivé en Chine». Le Parisien, 8 de agosto de 2008, en: https://www.leparisien.fr/sports/Jo/nicolas-sarkozy-est-arrive-en-chine-08-08-2008-128950.php; CAmBADÉLIS, Jean-Christophe: "Nicolas Sarkozy est partout, la France est nulle part, La Revue internationale et stratégique, 77, 2010, p. 78.

96. El PAís: «Sarkozy se reúne con el Dalai Lama pese a las amenazas de boicot de China», El País, 6 de diciembre de 2008, en: https://elpais.com/internacional/2008/12/06/actualidad/1228518013_850215.html 
MANUEL DE JESÚS ROCHA PINO

EL DESARROLLO DE LA RELACIÓN CHINA-FRANCIA: DEL RECONOCIMIENTO MUTUO 293 A LA PROYECCIÓN DE LA INICIATIVA CINTURÓN Y RUTA DE LA SEDA

China-UE ${ }^{97}$. La postergación de la Cumbre fue un hecho sorpresivo, pues hasta ese momento las advertencias de China solo hacían referencia al comportamiento de las autoridades francesas y a su impacto en la relación bilateral. En este caso, la sanción de China involucró a la agenda de su relación bilateral con la UE. Al respecto, el Portavoz del Ministerio de Asuntos Exteriores chino, Qin Gang, anunció:

Con el fin de mantener buenas relaciones con Francia y con la Unión Europea, China ha comunicado reiteradamente a Francia sobre la necesidad de manejar adecuadamente el asunto del Tíbet, con el fin de haber creado las condiciones necesarias para la cumbre China-uE. Por desgracia, la parte francesa no respondió activamente a los esfuerzos de China para el mantenimiento de las relaciones con Francia y la Unión Europea ${ }^{98}$.

Para Jing Men, la postergación de la cumbre China-uE en 2008 pudo interpretarse no solo como el inicio de una nueva etapa de las relaciones entre China y la UE sino incluso como una coyuntura que señaló un cambio en la política exterior china en general instrumentada durante el gobierno de Hu Jintao ${ }^{99}$. Desde el punto de vista chino, los inconvenientes eran responsabilidad del gobierno francés. En ocasión de la entrevista de Sarkozy con el Dalái Lama, un académico chino criticó:

Sarkozy sacrificó las relaciones de la uE con China, lo cual es definitivamente inconsistente con el objetivo de relacionarse con China [...] Si China hiciera diferentes políticas de acuerdo con los diferentes Estados miembros, no habría una uE con la cual relacionarse, sin importar la existencia de una política de China hacia la uE ${ }^{100}$.

Igualmente, la coyuntura de crisis diplomática extendida a la relación China-UE contribuyó a la paralización del diálogo bilateral durante algunos meses, en un momento inmediato al inicio de la crisis económica mundial de 2008, tras el derrumbe de los mercados financieros en septiembre; como comenta Liu Mingli:

[...] desde el otoño de 2008 la crisis financiera mundial que paralizó a Occidente de manera inesperada ha profundizado las incertidumbres de China respecto al papel que ejercen estos actores internacionales y su influencia, generando debates continuos en China sobre su política exterior ${ }^{101}$.

97. Consejo Europeo: Postponement of the Eu/China Summit. Statement by the European Union 16352/08 (Presse 347). Bruselas, 26 de noviembre de 2008, en: https://ec.europa.eu/commission/ presscorner/detail/en/PRES_08_347

98. QIN, Gang: "China postpones Summit with Eu due to French leader's planned meeting with Dalai Lama", Beijing Review, 27 de noviembre de 2008, en: http://www.bjreview.com/quotes/txt/200811/27/content_168605.htm

99. JING, Men: "The EU and China: Mismatched Partners?», Journal of Contemporary China, 24(71), 2012, p. 347.

100. Liu, Mingli: "Reflection on the UE-China Relations", Contemporary International Relations (Pekín), 30(3), 2010, p. 118.

101. Idem. 


\section{MANUEL DE JESÚS ROCHA PINO \\ EL DESARROLLO DE LA RELACIÓN CHINA-FRANCIA: DEL RECONOCIMIENTO MUTUO A LA PROYECCIÓN DE LA INICIATIVA CINTURÓN Y RUTA DE LA SEDA}

La 11 cumbre China-uE pudo realizarse en mayo de 2009 en Praga, durante la presidencia rotatoria del Consejo encabezada por la República Checa. De manera significativa, en ocasión de la 11 Cumbre se redefinieron las nuevas prioridades en la relación bilateral sino-europea, ahora en el contexto de la grave crisis económica iniciada el año anterior: con esta redefinición, las prioridades de la agenda sino-europea se enfocaron en la profundización de las relaciones económico-comerciales de una manera pragmática, mientras la agenda normativa de la buena gobernanza quedaba subordinada al interés económico.

La suspensión de la Cumbre China-uE en 2008 sirvió, desde la perspectiva china, como una reafirmación de sus prioridades. Una vez que inició la nueva Presidencia del Consejo en 2009, Pekín realizó los acercamientos para la realización de la Cumbre. En el Comunicado Conjunto de la 11 Cumbre se enfatizó:

$\mathrm{Al}$ iniciar una nueva década para la Cumbre uE-China, las dos partes reafirmaron su firme compromiso de lograr la asociación estratégica integral uE-China y su voluntad de trabajar juntos para su desarrollo mutuo, con una visión de futuro basada en los principios de respeto mutuo, igualdad, confianza, reciprocidad y cooperación ${ }^{102}$.

De acuerdo con Jean-Pierre Cabestan, se pueden identificar cuatro aspectos que guiaron a la política exterior francesa hacia China durante el gobierno de Sarkozy:

a) Francia adquirió la concepción de que China debía ser un actor más responsable conforme adquiría poder e influencia en el sistema internacional: especialmente en cuestiones económico-comerciales, así como en las áreas referentes a la política, los derechos humanos o la protección del medio ambiente ${ }^{103}$.

b) El gobierno de Sarkozy se mostró renuente para otorgar a China un estatus preferente especial, desplazada por otras prioridades en la agenda de la política exterior francesa como Rusia o fue equiparada en importancia a otras economías asiáticas como India o Japón: Sarkozy prefirió calificar las relaciones con China como una asociación estrecha o cercana (parteneriat étroit) en lugar de una asociación estratégica global (perteneriat stratégique global) ${ }^{104}$.

Esta unilateral «rectificación del nombre» de la asociación provocó malestar en Pekín. En la Declaración Conjunta de la asociación estratégica global de 2004 se afirmaba:

La ampliación de la asociación estratégica global franco-china se encuentra ahora en el corazón de la relación euro-china, cuyo desarrollo constituye un

102. Consejo Europeo: 11th eu-China Summit Prague, 20 May 2009 Joint Press Communiqué. Praga, 20 de mayo de 2009, en: https://ec.europa.eu/clima/sites/clima/files/docs/0023/ joint_statement_en.pdf

103. Cabestan, Jean-Pierre, op. cit. p. 137

104. Idem. 


\section{MANUEL DE JESÚS ROCHA PINO \\ EL DESARROLLO DE LA RELACIÓN CHINA-FRANCIA: DEL RECONOCIMIENTO MUTUO 29) A LA PROYECCIÓN DE LA INICIATIVA CINTURÓN Y RUTA DE LA SEDA}

elemento importante en la escena internacional. Francia y China contribuirán al fortalecimiento de esta relación en todos los ámbitos ${ }^{105}$.

Es decir, entre 2008-2009, la aspiración francesa por ocupar un lugar central en las políticas europeas hacia China igualmente trasmitió una coyuntura de crisis a la relación China-uE, al menos durante corto tiempo.

c) El gobierno de Sarkozy se orientó de manera más firme en el principio de reciprocidad como base de la relación bilateral, asumiendo una postura más realista que la de Chirac, además de tener como prioridad a la promoción universal de los derechos humanos, algo que terminó afectando a la relación sino-francesa ${ }^{106}$. Durante el periodo de Sarkozy perdieron relevancia los contactos bilaterales de alto nivel (básicos para instrumentar la agenda de la asociación estratégica), mientras París demostró una preferencia por el multilateralismo como escenario para los acercamientos entre Francia y China, especialmente durante los llamados a establecer medidas de coordinación para enfrentar la crisis económica.

Sin embargo, los términos de las prioridades económicas, de manera pragmática, quedaron esclarecidos durante la visita de Hu Jintao a Francia en noviembre de 2010: en la misma, Sarkozy aludió a las relaciones de cooperación y "los logros de nuestra asociación global estratégica" "les réussites de notre partenariat global stratégique»), al mismo tiempo que Hu mantuvo una reunión privada con el expresidente Chirac ${ }^{107}$. Posteriormente, durante la reunión que Hu Jintao y Sarkozy sostuvieron en noviembre de 2011, en ocasión de la Sexta Cumbre del G-20 en Cannes, el presidente francés solicitó una mayor inversión de las reservas internacionales de China en los bonos de deuda soberana de los países europeos más endeudados como Grecia y Portugal: el gobierno chino señaló que «apoyaba al proceso de integración europeo» pero que también "le correspondía a Europa resolver el problema de la deuda soberana ${ }^{108}$. Al final del gobierno de Sarkozy se había impuesto la racionalidad del pragmatismo económico como una característica estructural que se ha mantenido en la relación China-ue desde 2009 hasta la actualidad (a pesar de las incertidumbres propias de la crisis de 2020); sin embargo, el cambio en las percepciones de Sarkozy no fue suficiente para reconstruir la confianza entre Pekín y París: le correspondería al siguiente gobierno francés instrumentar las medidas para normalizar la relación con China.

105. Gobierno de la REPública Francesa, 2004, op. cit.

106. CABESTAn, Jean-Pierre, op. cit. p. 137

107. Presidencia de la RePública francesa: Déclaration de M. Nicolas Sarkozy, Président de la République, sur les relations franco-chinoises, à Paris le 4 novembre 2010. París, 4 de noviembre de 2010, en: https://www.vie-publique.fr/discours/180364-declaration-de-m-nicolas-sarkozy-presidentde-la-republique-sur-les-r; MINISTERIO DE ASUNTOS EXTERIORES DE CHINA: Hu Jintao holds talks with his French Counterpart Sarkozy. París, 5 de noviembre de 2010, en: https://www.fmprc.gov.cn/mfa_eng/ topics_665678/hujintaofangwenfaguoheputaoya_665778/t767230.shtml

108. Deutschewelle: "China ReluctanttofundEurozoneBailout", Deutsche Welle, Cannes, 31deoctubre de 2011, disponible en: http://www.dw.com/en/china-reluctant-to-fund-eurozone-bailout/a-15499683 
MANUEL DE JESÚS ROCHA PINO

EL DESARROLLO DE LA RELACIÓN CHINA-FRANCIA: DEL RECONOCIMIENTO MUTUO

A LA PROYECCIÓN DE LA INICIATIVA CINTURÓN Y RUTA DE LA SEDA

5. La relación China-Francia durante los periodos de Gobierno de François Hollande (2012-2017) y Emmanuel Macron (2017) y la PROYección de la Ruta de la Seda Marítima del Siglo XXI

La relación sino-francesa durante la presidencia de François Hollande estuvo determinada desde un inicio por la crisis económica. Las relaciones comerciales y de inversión se convirtieron en los temas principales de la agenda, teniendo a los llamados del gobierno francés por mantener una relación económica «equilibrada» con China el aspecto que pudiera generar una mayor tensión ${ }^{109}$ : para incentivar los flujos comerciales y de inversión, así como la reactivación de la agenda de cooperación en la asociación estratégica bilateral, Hollande recurrió a la diplomacia de cumbres de alto nivel (descuidada durante el periodo de Sarkozy). Desde 2009 los temas normativos en la agenda habían quedado subordinados a las prioridades económicas, de manera similar a lo ocurrido en la relación China-uE.

Hollande realizó su primera visita oficial a China en abril de 2013, una de sus primeras acciones en Pekín fue reafirmar la «importancia de la asociación estratégica" firmada en 1997 y la renovada "asociación global estratégica"» de 2004, al mismo tiempo que convocaba a iniciar a una nueva etapa de los términos de la asociación (el cuidado de Hollande al describir los mecanismos de cooperación con China probablemente se debió al tropiezo provocado por Sarkozy al tratar de redefinir la terminología oficial) ${ }^{110}$. En su discurso, Hollande enfatizó las prioridades de la agenda como:

[...] la emergencia de un mundo multipolar, la profundización de los intercambios económico-comerciales a través de una asociación de beneficio mutuo (ganar-ganar) y la profundización de los intercambios culturales ${ }^{111}$.

En 2011 el déficit comercial desfavorable a Francia había ascendido a 27.6 mil millones de euros (alrededor del $40 \%$ de su déficit total), una evidencia de que en lo comercial la relación se desarrollaba de una manera "asimétrica y desequilibrada». Además de normalizar los contactos de cooperación, Hollande tuvo como prioridad una modificación del ritmo de crecimiento del déficit comercial con China que se había duplicado en menos de diez años ${ }^{112}$. Desde la perspectiva de Pekín, la aproximación en el inicio del gobierno de Hollande se podría definir

\footnotetext{
110. Heng-Lim, Yves, op. cit. p. 29. Presidencia de la república francesa: Déclatation de mm. François Hollande, Président de la République, et Xi Jinping, président de la République populaire de Chine, sur les relations franco-chinoises, à Pékin le 25 avril 2013. Pekín, 25 de abril de 2013, en: https:// www.elysee.fr/front/pdf/elysee-module-12714-fr.pdf

111. Idem.

112. Heng-Lim, Yves, op. cit. pp. 30-31.
} p. 157.

109. Lincot, Emmanuel: «Réinventer les relations franco-chinoises», Géoéconomie, 67, 2013, 
MANUEL DE JESÚS ROCHA PINO

EL DESARROLLO DE LA RELACIÓN CHINA-FRANCIA: DEL RECONOCIMIENTO MUTUO 297 A LA PROYECCIÓN DE LA INICIATIVA CINTURÓN Y RUTA DE LA SEDA

como una «diplomacia económica», una percepción que continuó durante su presidencia ${ }^{113}$.

Una consecuencia de la relación sino-francesa desarrollada durante el periodo de Sarkozy era la certeza de que el tiempo de la «relación privilegiada» entre Pekín y París pertenecía al pasado ${ }^{114}$ : en el contexto de la relación China-uE, era el gobierno francés quien adoptaba las prioridades de Bruselas, orientadas hacia el pragmatismo económico, sin existir mucha evidencia sobre una transmisión de las preferencias de Francia a la uE en la materia (mientras el déficit comercial era la muestra tangible de la pérdida de la "relación privilegiada») ${ }^{115}$. Al nuevo contexto también se le puede atribuir la reorganización de las Cumbres China-uE como consecuencia de la entrada en vigor del Tratado de Lisboa en 2009: a partir de 2010, las Cumbres sino-europeas fueron coordinadas por las autoridades de la Comisión, el Consejo y el Alto Representante para Asuntos Exteriores y Política de Seguridad y no por la Presidencia rotatoria del Consejo de la UE. La penúltima cumbre coordinada por la Presidencia rotatoria del Consejo habría sido la de Lyon realizada posteriormente en Praga (a continuación, la 12 Cumbre China-ue se realizó en Nanjing en noviembre de 2009). Por otra parte, al iniciar el gobierno de Hollande, el acuerdo de cooperación referencial en la relación sino-europea era la Agenda de Cooperación China-ue de 2013, redactada de manera conjunta entre el Ministerio de Asuntos Exteriores de China y el Servicio Europeo de Acción Exterior.

En marzo de 2014, Xi Jinping realizó una visita oficial a Francia en ocasión de la conmemoración del 50 aniversario del establecimiento de las relaciones diplomáticas: como parte de su viaje, visitó Lyon para presentar los primeros proyectos de la Iniciativa Cinturón y Ruta de la Seda en Francia (el ferrocarril Wuhan-Lyon se inauguraría en 2016); de una manera simbólica, Xi comentó:

Lyon fue uno de los puntos importantes de llegada a Europa para la Ruta de la Seda, cuyo punto de partida era Shaanxi, mi provincia natal [...] También es en Lyon donde todos los líderes del Partido Comunista Chino de la vieja generación como Deng Xiaoping, Chen Yi, Nie Rongzhen y Cai Hesen hicieron una estancia [como trabajadores entre 1919-1921] ${ }^{116}$.

Igualmente, el Presidente Xi continuó su visita por Alemania y Bélgica con la finalidad de presentar los primeros proyectos de la Iniciativa Cinturón y Ruta: al

113. China DAILY: "President Xi calls on China, Germany to build Silk Road Economic Belt", China Daily, 30 de marzo de 2014, en: https://www.chinadaily.com.cn/world/2014xivisiteu/201403/30/ content_17389965.htm

114. Courmont, Barthélemy y Lincot, Emmanuel: «La relation diplomatique Franco-chinoise à l'horizon 2020", Monde Chinois, 32, 2012, p. 11.

115. Cubizol, Damien et. al: "Le commerce franco-chinois: leçons du passé et perspectives d'avenir", Monde Chinois, 59, 2019, pp. 37-38.

116. AFP: "Le président chinois entame à Lyon sa visite en France», L'Express, 25 de marzo de 2014, en: https://www.lexpress.fr/actualites/1/societe/le-president-chinois-entame-a-lyon-sa-visite-enfrance_1503078.html *Cursivas agregadas por el autor. 
parecer la prioridad comercial de China eran Alemania (específicamente Duisburgo, en donde culmina la ruta del ferrocarril Yuxinou, Chongqing-Xinjiang-Duisburgo) y la sede de la ue en Bruselas ${ }^{117}$. Posteriormente, en 2015, París aceptó la invitación para participar como uno de los países fundadores del Banco Asiático de Inversión en Infraestructura en una acción pragmática que provocó tensiones y críticas por parte del gobierno de Barack Obama: a pesar de las advertencias estadounidenses que disuadían la membresía de los países de la uE en el BAII, el Reino Unido fue el primero en unirse en marzo de 2015. Tras la decisión de Londres, le siguieron Francia, Alemania e Italia (entre los miembros del G7) ${ }^{118}$.

Durante el resto del gobierno de Hollande existió una continuidad de la diplomacia de cumbres realizadas de maneral anual. Además, conforme los efectos de la crisis comenzaban a ser superados en la uE (el turismo de ciudadanos chinos en Francia adquiría una mayor importancia), a partir de 2016, se clarificaron los proyectos de inversión de China en Francia como parte de la Ruta de la Seda Marítima del siglo 21 (una vertiente de la Iniciativa Cinturón y Ruta): una de las prioridades ha sido la inversión en el Puerto de Marsella en concordancia con las inversiones realizadas por China en otros puertos del Mediterráneo como Valencia y el Pireo en Grecia. El objetivo del capital chino ha sido diversificar los espacios de contacto entre el Cinturón Económico de la Ruta de la Seda con la Ruta de la Seda Marítima que tiene como espacio geopolítico a la costa del Mediterráneo; específicamente en Marsella sobresalen dos proyectos de inversión: el proyecto MIF 68 (Marseille International Fashion Center), una zona mayorista y semi-mayorista de distribución de productos textiles que incluye a grupos de importadores y el proyecto de inversiones de la planta de Quechen Silicon Chemical ${ }^{119}$. De acuerdo con el Director de Quechen, Que Weidong:

Marsella tiene la ubicación perfecta con gran acceso a Europa y el norte de África y tiene todo lo que necesitamos en términos de recursos: agua, gas, electricidad, tierra disponible [...] Si el Mediterráneo es una corona, entonces Marsella es la perla superior ${ }^{120}$.

Los proyectos de inversión también incluyen a las industrias digitales debido a que el área metropolitana de Aix-Marseille es uno de los cinco principales

117. China Daily: "President Xi calls on China, Germany to build Silk Road Economic Belt", China Daily, 30 de marzo de 2014, en: https://www.chinadaily.com.cn/world/2014xivisiteu/2014-03/30/content_17389965.htm

118. Dieng, Moda y Liu, Bingyang: "Les enjeux de l'expérience chinoise en matière de multilatéralisme: le cas de la Banque asiatique d'investissement dans les infrastructures", Études internationales, 46(4), 2015, p. 532.

119. Belguidoum, Saïd y Souinh, Farida: «Les nouvelles routes de la soie en Méditerranée», Confluences Méditerranée, 109, 2019, p. 12.

120. Yuan, Shenguo: "Country's Businesses, like its Tourists, flocking to French Provence Región", China Daily, 26 de marzo de 2019, en: http://www.chinadaily.com.cn/cndy/2019-03/26/content_37451751.htm 
MANUEL DE JESÚS ROCHA PINO

EL DESARROLLO DE LA RELACIÓN CHINA-FRANCIA: DEL RECONOCIMIENTO MUTUO 29) A LA PROYECCIÓN DE LA INICIATIVA CINTURÓN Y RUTA DE LA SEDA

centros de telecomunicaciones de Europa y cuenta con la mejor infraestructura de almacenamiento de datos de su clase (la región es uno de los principales centros de inversión de China Telecom, al que se suma Mónaco luego del Memorando de Entendimiento entre Huawei y Monaco Telecom de 2019). Igualmente, en 2018, el Puerto de Shanghai acordó un esquema de cooperación con el Puerto de Marsella. El compromiso de cooperación quedó clarificado durante la Conferencia de la Iniciativa Cinturón y la Ruta de 2018 en Marsella, con la asistencia del ex Primer Ministro Jean-Pierre Raffarin, Zhai Jun, el embajador de China en Francia, y Fei Zhaohui, presidente de la sucursal en París del Banco de Exportaciones e Importaciones de China (China's Exim Bank) ${ }^{121}$.

En un inicio, la presidencia de Emmanuel Macron mostró una continuidad con el gobierno de Hollande, al menos durante el periodo 2017-2019, aunque mostrando un mayor interés por establecer nexos de cooperación con las inversiones de capital chino en la UE. Sin embargo, el gobierno francés también ha mostrado cierto escepticismo en temas como el déficit comercial bilateral, las inversiones de China en activos industriales en la uE y la diversificación de las iniciativas de cooperación de China en otros espacios de Europa como en los casos del Foro de Cooperación China-Europa Central y Oriental (Foro 17+1) y el Memorando de Entendimiento China-Italia de 2019 ${ }^{122}$. En 2019 el intercambio comercial sino-francés ascendió a 73.4 mil millones de euros, aunque con un déficit desfavorable a Francia de 31.6 mil millones (más de la mitad del déficit total del comercio francés de 58.9 mil millones): sin embargo, durante la visita oficial de Xi a Francia en 2019, Pekín anunció acuerdos comerciales e inversiones por 40 mil millones de euros en territorio francés. En 2020 el déficit comercial sino-francés alcanzó un récord de 38.9 mil millones de euros: «este incremento se explica por el aumento de las importaciones de material médico» desde China debido a la pandemia Covid-19 ${ }^{123}$.

Al respecto del Memorando de Entendimiento China-Italia, acordado durante la visita de Xi Jinping a Roma en marzo de 2019, el Presidente Macron realizó críticas al gobierno italiano por su acción «ingenua» al firmar un acuerdo que lo podría hacer dependiente de las inversiones del capital chino y apoyó una redefinición de la relación China-uE en los términos de un "competidor económico» y

121. Idem.

122. Zhang, Ji: "France's Policy towards China in 2017». En: vvaA. China Policies of the Eu and its Members (2017): Two Patterns of Differentiated Cooperation. Shanghai: Center for China-Eu Relations, Fudan University, Institute for European Studies, 2017, pp. 20-22, en: http://www.sies-cn.org/UploadFiles/file/european\%20survey/201803210441245878_China\%20Policies\%20of\%20the\%20Eu\%20and\%20 Its\%20Members\%20(2017)(1).pdf.pdf

123. MiNISTERIO DE ECONOMÍA, FINANZAS y RECUPERACIÓN DE FRANCIA: Echanges bilatéraux entre la France et la Chine, 17 de mayo de 2021, en: https://www.tresor.economie.gouv.fr/Pays/CN/echangesbilateraux-entre-la-france-et-la-chine; FrANce 24: «France, China sign Multibillion Trade Deals as Xi Jinping meets Macron", France 24, 25 de marzo de 2019, en: https://www.france24.com/en/20190325france-china-sign-multibillion-trade-deals-xi-jinping-meets-macron; DEuTSCHE welle: "Xi, Merkel, Macron and Juncker meet in Paris", Deutsche Welle, 26 de marzo de 2019, en: https://www.dw.com/en/ xi-merkel-macron-and-juncker-meet-in-paris/a-48060985 
MANUEL DE JESÚS ROCHA PINO

EL DESARROLLO DE LA RELACIÓN CHINA-FRANCIA: DEL RECONOCIMIENTO MUTUO

A LA PROYECCIÓN DE LA INICIATIVA CINTURÓN Y RUTA DE LA SEDA

«rival sistémico» formulada en un Documento Estratégico sobre China de la Comisión Europea: durante su estancia en París, el Presidente Xi sostuvo una reunión cuatripartita con Macron, con la Canciller Merkel y el entonces Presidente de la Comisión Europea, Jean Claude Juncker, en la que se consiguió disminuir la tensión diplomática ${ }^{124}$. Posteriormente, durante la 21 Cumbre China-uE realizada en Bruselas, China y la uE acordaron eliminar las prácticas de transferencia forzosa de tecnología para los activos industriales europeos adquiridos por empresas de capital chino ${ }^{125}$.

En los aspectos geopolítico y geoeconómico, París compartió algunas preferencias con la política exterior estadounidense del gobierno de Donald Trump, al apoyar su discurso sobre un "Indo-Pacífico libre y abierto", aunque rechazó los términos del proteccionismo y su guerra comercial, manteniendo una posición equidistante entre los intereses comerciales con China y los compromisos estratégicos con exuu ${ }^{126}$. Como muestra, el gobierno francés ha resistido sumarse al veto inmediato en contra de las empresas tecnológicas chinas y su tecnología $5 \mathrm{G}$ exigido por Trump a los miembros de la UE; sin embargo, en julio de 2020, París anunció que buscaba eliminar toda la infraestructura de las redes $5 \mathrm{G}$ operada por las empresas tecnológicas chinas teniendo como límite el año 2028: una decisión dirigida a disminuir las tensiones con Washington mientras proporcionaba a las autoridades francesas un amplio margen de tiempo para sustituir a la tecnología digital de empresas como Huawei ${ }^{127}$.

La política exterior francesa hacia China, durante los gobiernos de Hollande y Macron, mostró aceptar los términos estructurales del pragmatismo económico concretado, a su vez, entre Pekín y Bruselas, si bien durante el periodo de Macron comenzaron a manifestarse los primeros efectos de la competición geopolítica instrumentada por Trump hacia China (en un momento contextualizado por la crisis mundial provocada por la propagación de la enfermedad Covid-19). A partir de la reestructuración de la relación transatlántica propuesta por el gobierno de Joseph Biden en 2021, que implica la coordinación de eEuU con los miembros de la uE en la implementación activa de políticas de contención económica frente a China, en principio el gobierno de Macron ha mantenido una postura equilibrada entre los compromisos con la alianza occidental y la continuación de las relaciones económico-comerciales con China, además de la agenda de la cooperación en materia

124. Idem

125. Consejo europeo: 21 eu-China Summit Joint Statement. Bruselas, 9 de abril de 2019, en: https://www.consilium.europa.eu/media/39020/euchina-joint-statement-9april2019.pdf

126. France 24: "France challenges Beijing in South China Sea", France 24, 12 de junio de 2018 , en: https://www.france24.com/en/20180612-france-challenges-beijing-south-china-sea

127. Reuters: "Exclusive: French limits on Huawei 5G Equipment Amount to de facto ban by 2028", Reuters, 22 de julio de 2020, en: https://www.reuters.com/article/us-france-huawei-5g-security-exclusive/ exclusive-french-limits-on-huawei-5g-equipment-amount-to-de-facto-ban-by-2028-iduSKCN24N26R 
MANUEL DE JESÚS ROCHA PINO

EL DESARROLLO DE LA RELACIÓN CHINA-FRANCIA: DEL RECONOCIMIENTO MUTUO 301 A LA PROYECCIÓN DE LA INICIATIVA CINTURÓN Y RUTA DE LA SEDA

medioambiental y para el desarrollo en África (en el contexto de la recuperación económica post-Covid) ${ }^{128}$.

\section{CONClusiones}

En la investigación se analizaron los principales aspectos estructurales desarrollados por la relación China-Francia desde que ambas establecieron relaciones diplomáticas en 1964. Como un primer elemento se identificó a la importancia de las políticas de reconocimiento mutuo entre París y Pekín, tanto para que Francia desarrollara una relación "privilegiada" y cercana con China, de una manera que superara a sus socios europeos, como en el interés de China para relacionarse con un país relevante en Europa como Francia. La importancia de la política de reconocimiento mutuo se evidenció durante el proceso de negociación para oficializar las relaciones diplomáticas: así fue en el caso de la ruptura francesa con Taiwán y su reconocimiento de la política de «una sola China». Igualmente, la importancia de las relaciones económico-comerciales fue un incentivo definitivo para el establecimiento de las relaciones.

Por otra parte, la posición de Francia como interlocutor de China en la CEE contribuía para que el gobierno francés mantuviera el objetivo de trasmitir sus preferencias hacia las políticas europeas hacia China (una condición que se mantuvo hasta el periodo de gobierno de Sarkozy y el contexto de crisis económica mundial). Desde un inicio los términos de la relación estuvieron determinados por los intereses nacionales de ambos países, quienes podían aproximarlos en algunas coyunturas, e incluso en oposición a las preferencias de sus propios aliados (aunque de una manera limitada y sin cuestionar el statu quo).

Durante el resto de la Guerra Fría los gobiernos franceses se centraron en continuar con su percepción de mantener la relación "privilegiada" con China aunque con el establecimiento generalizado de relaciones diplomáticas entre Pekín y los países de Europa occidental, durante la década de 1970, la economía china comenzó a diversificar sus relaciones con los miembros de la CEE: de esta manera, Alemania alcanzó a situarse como el primer socio comercial de China en Europa superando a la economía francesa. Durante el gobierno de Mitterrand se mantuvo el rasgo estructural de la relación privilegiada y la importancia de las relaciones económicas; sin embargo, a partir de 1989, la agenda normativa (relacionada con los valores de la buena gobernanza como los derechos humanos) irrumpió en la relación sino-francesa para determinar su rumbo en los siguientes años.

128. Ver: PRESIDENCIA DE LA REPÚBLICA FRANCESA: Entretien en visioconférence avec la Chancelière de la République fédérale d'Allemagne, Mme Angela MERKEL, et le Président de la République populaire de Chine, M. xi Jinping. París, 5 de julio de 2021, en: https://www.elysee.fr/emmanuel-macron/2021/07/05/ entretien-en-visioconference-avec-la-chanceliere-de-la-republique-federale-dallemagne-mme-angelamerkel-et-le-president-de-la-republique-populaire-de-chine-m-xi-jinping 
La prioridad del gobierno francés alrededor de la agenda normativa se instrumentó en un contexto de crisis interna en China provocada por la represión militar en contra de la población, especialmente en la Plaza Tiananmen, debido a las manifestaciones de la población en contra de la corrupción y en favor de la democracia. Como consecuencia, Francia encabezó las políticas de sanciones económicas en contra de Pekín en la CEe que con el tiempo resultaron dañinas para los propios intereses de Francia y deterioraron de manera importante la relación bilateral: al final del periodo de Mitterrand, entre 1994-1995, el gobierno francés propuso una aproximación a Pekín en términos pragmáticos.

El segundo aspecto estructural, identificado en la investigación, son los términos pragmáticos de las relaciones económicas, siendo el nuevo gobierno francés, encabezado por Jacques Chirac, el encargado de instrumentar plenamente dicha agenda. La presidencia de Chirac retomó las prioridades definidas durante el gobierno de de Gaulle en cuanto a privilegiar las relaciones económicas y continuar con la percepción de la relación "privilegiada" (al asumir discursos como el de la multipolaridad y al establecer una asociación estratégica bilateral en 1997). Sin embargo, de manera paradójica, la continuidad de la agenda de la relación sinofrancesa, y la transmisión efectiva de sus preferencias a las políticas de la uE hacia China, a pesar de las diversas actividades de cooperación, tuvo una consecuencia solamente coyuntural: en pocos meses, el sucesor de Chirac en la presidencia, Nicolas Sarkozy, implementó una nueva aproximación hacia China que (temporalmente) dejó sin efecto al legado del gobierno anterior.

Quizá como respuesta a la aproximación que había tenido la relación sinofrancesa en 2003, al oponerse de manera consensuada a la invasión estadounidense a Irak, el gobierno de Sarkozy se inclinó por acercar sus preferencias a las estadounidenses y hacer un mayor énfasis en la agenda normativa (especialmente en cuanto a los señalamientos sobre la situación de los derechos humanos en China). Además del deterioro de la relación, una consecuencia no planeada para el gobierno de Sarkozy fue la pérdida de la capacidad de Francia para influir en las políticas comunitarias hacia China (una situación que se consolidó con los cambios institucionales, como la puesta en vigor del Tratado de Lisboa en 2009, y con el inicio de la crisis económica mundial).

La relación sino-francesa inició un proceso de normalización y activación de la agenda de la asociación estratégica durante los gobiernos de Hollande y Macron siendo el pragmatismo económico el principal rasgo estructural que ha prevalecido hasta el momento (algo que favoreció la recepción inicial en Francia de la Iniciativa Cinturón y Ruta de la Seda). A partir de 2019, el gobierno de Macron asumió una posición más realista y crítica hacia las actividades comerciales y de inversión de China en la UE, aunque manteniendo (todavía) la racionalidad pragmática en la relación bilateral. 